\title{
Time-restricted feeding promotes skeletal muscle function in diet- and genetic-induced obesity through shared and unique pathways
}

\section{Christopher Livelo}

University of Alabama Birmingham School of Medicine

\section{Yiming Guo}

University of California Riverside

\section{Shweta Varshney}

Salk Institute for Biological Studies

\section{Farah Abou Daya}

University of Alabama Birmingham School of Medicine

\section{Hiep Le}

Salk Institute for Biological Studies

\section{Satchidananda Panda}

The Salk Institute for Biological Studies

Girish Melkani ( $\square$ gmelkani@uab.edu )

University of Alabama Birmingham School of Medicine https://orcid.org/0000-0003-0353-4633

\section{Article}

Keywords: Time-restricted feeding, diet-induced obesity, genetic-obesity, circadian rhythm, insulin resistance and sensitivity, Drosophila genetics, skeletal muscle physiology, metabolic homeostasis, triglycerides, obesogenic challenges, purine synthesis, AMPK-signaling, TCA, OxPhos, ETC, glycolysis, glycogen metabolism

Posted Date: September 8th, 2021

DOI: https://doi.org/10.21203/rs.3.rs-828135/v1

License: (c) (i) This work is licensed under a Creative Commons Attribution 4.0 International License. Read Full License 


\section{Time-restricted feeding promotes skeletal muscle function in diet- and genetic-induced obesity through shared and unique pathways}

Christopher Livelo ${ }^{1 *}$, Yiming Guo ${ }^{1 *}$, Shweta Varshney ${ }^{2,3}$, Farah Abou Daya ${ }^{1}$, Hiep D. Le ${ }^{3}$, Satchidananda Panda ${ }^{3}$, Girish C. Melkani ${ }^{1,2 \dagger}$

${ }^{1}$ Department of Pathology, Division of Molecular and Cellular Pathology, School of Medicine, The University of Alabama at Birmingham, AL 35294, USA.

2Department of Biology and Molecular Biology Institute, San Diego State University San Diego, CA 92182, USA.

${ }^{3}$ Regulatory Biology Laboratory, Salk Institute for Biological Studies, La Jolla, CA 92037, USA.

tCorresponding Address: Department of Pathology, Division of Molecular and Cellular Pathology, School of Medicine, University of Alabama at Birmingham, AL 35294, USA. Tel.: 1205-996-0591; Fax: 1-205-934-7447; E-mail: girishmelkani@uabmc.edu (GCM)

${ }^{*}$ Contributed equally 


\begin{abstract}
Obesity caused by genetic predisposition, a lifestyle of calorie-dense diets and/or circadian disruption can result in complications including metabolic syndrome, cardiovascular disease, and compromised muscle function. By employing time-restricted feeding (TRF), where daily feeding was limited to 12 hours during the day, we observed improved skeletal muscle function compared to ad libitum feeding (ALF). This was observed in both diet-induced obesity (DIO) and genetic-induced obesity (GIO) in a Drosophila melanogaster (fruit fly) model. We evaluated the mechanistic basis of TRF-mediated benefits by utilizing muscle transcriptomic data of indirect flight muscle (IFM) followed by genetic validations, cytological and biochemical evidences. Significant upregulation of glycine $N$ methyltransferase (Gnmt), sarcosine dehydrogenase (Sardh), CG5955 and downregulation of diacylglycerol o-acyltransferase 2 (Dgat2) were commonly induced by TRF intervention under both obese conditions. Moreover, genetic inhibition of Gnmt, Sardh and CG5955 leads to skeletal muscle dysfunction, aberrant lipid accumulation and loss of TRF-mediated benefits. However, skeletal musclespecific knockdown $(K D)$ of Dgat2 retained muscle function during aging, a result that mimics TRFmediated benefits. Furthermore, de novo purine biosynthesis appeared to be upregulated specifically in the DIO model under TRF which led to increased ATP levels resulting in improved muscle performance. Additionally, genes associated with AMP kinase (AMPK) signaling, glycogen metabolism, glycolysis, tricarboxylic acid (TCA) cycle and electron transport chain (ETC) signaling were specifically upregulated in GIO model under TRF. TRF mediated benefits in GIO via activation of AMPK, which led to increased ATP levels. Altogether, we identify the shared and distinct pathways in the regulation of muscle function under TRF, which may aid further research and alternative therapeutic avenues that focus on combating comorbidities linked with obesity.
\end{abstract}

Keywords: Time-restricted feeding, diet-induced obesity, genetic-obesity, circadian rhythm, insulin resistance and sensitivity, Drosophila genetics, skeletal muscle physiology, metabolic homeostasis, triglycerides, obesogenic challenges, purine synthesis, AMPK-signaling, TCA, OxPhos, ETC, glycolysis, glycogen metabolism 


\section{Introduction:}

Obesity is a global and public health problem linked to various comorbidities ${ }^{1}$. Major contributors to obesity include living a lifestyle comprising high-caloric diets, having a genetic predisposition to the disease and disruption of circadian rhythms ${ }^{2,3}$. Pathological obesity also reduces skeletal muscle function potentially due to decreased muscle mass and quality leading to compromised locomotor capabilities and lower mobility ${ }^{4}$. The skeletal muscle plays a crucial role in glucose metabolism as it is the major tissue responsible for glucose uptake from the blood $^{5}$. Indeed, intramyocellular lipids or intramyocellular triglycerides (IMCL/IMTG) catalyzed by diacylglyceride acyltransferase (Dgat1/2) deposited within skeletal muscle cells can be harmful if not routinely depleted as observed in athletes ${ }^{6}$. Such accumulation of IMCL/IMTG may lead to insulin resistance and muscular dysfunction ${ }^{7}$. Additionally, truncal adiposity has been associated with increased levels of $s$-adenosylmethionine (SAM) in overfed humans ${ }^{8}$. SAM is a universal methyl donor involved in various physiological processes and increased levels have been observed to be a pathogenic catalyst which requires regulation from entities such as glycine $N$ methyltransferase (Gnmt). GNMT converts SAM levels to sarcosine with the help of glycine, a product from reactions involving CG5955 and sarcosine dehydrogenase (Sardh) ${ }^{9}$.

In addition to diet and genetics, the disruption of natural circadian rhythms is surfacing as an additional determinant for risk of obesity and associated metabolic disease ${ }^{3}$. Methods that perturb circadian rhythms through indirect and direct mechanisms have been found to have harmful effects on regulators involved in optimizing nutrient sensing, metabolism and energy availability leading to metabolic consequences $^{10,11}$. Despite oscillations of circadian rhythm being based on self-autonomous regulation, the synchronous workings of the circadian oscillators rely on external environmental cues which include light-dark periodicity, sleep, and timing of feeding ${ }^{12}$. Due to the reliance and importance of external cues, aberrant changes in patterns of these factors can have metabolically damaging consequences which potentially predispose individuals to metabolic dysfunction and elevate risks of obesity associated phenotypes.

Interestingly, interventions such as time-restricted feeding (TRF) help maintain homeostasis of feeding rhythmicity without any alteration of the total caloric intake. As a result, TRF regulates gene expression, maintains functionality and gene rhythmicity which leads to amelioration of obesity and metabolic dysfunction ${ }^{11,13}$. A Recent study with 11 obese men in a randomized cross-over design demonstrated in skeletal muscle that short-term TRF was sufficient to modulate rhythmic metabolism of lipids and amino acids and improve nocturnal glucose levels and insulin profiles during the day ${ }^{14,15}$. Another study utilizing 22 physically active college men under a 28-day TRF protocol displayed significant reduction in blood pressure, increased HDL-c and an increase in adiponectin plus lowered body fat percentage and mass ${ }^{16}$. These human studies taken together indicate that interventions such as TRF are potentially impactful in managing pathologies related to metabolism and obesity while providing a natural and affordable form of alternate therapy. TRF is also beneficial in animal models of obesity, ameliorating dysfunction in mouse liver and Drosophila heart and muscle ${ }^{11,13,17}$. However, there is little information regarding the mechanistic impacts of TRF on skeletal muscle in different obesity models.

Drosophila is an amenable model for studying human metabolic disease as mechanisms associated with nutritional sensing, energy utilization and energy storage are all conserved ${ }^{18}$. Additionally, Drosophila muscular organization regarding aging and disease is well established and is highly relevant to studying molecular and mechanistic workings ${ }^{19,20}$. We have previously used obese Drosophila in either a diet-induced obesity (DIO) or genetic-induced obesity (GIO) model ${ }^{11}$. In the DIO model, excess fat was added to fruit fly diet to induce obesity and its associated comorbidities as seen in other animal models and humans ${ }^{18-20}$. Furthermore, lethality can be observed in mice models with homozygous sphingosine kinase-2 (SK2), which is not lethal in Drosophila indirect flight muscle (IFM) specific $K D$ models allowing the study of muscular degeneration in more genes ${ }^{21}$. In the GIO model, 
Drosophila lacking Sk2 was utilized for studying genetic related obesity as mutations within Sk2 have been shown to result in ceramide accumulation also seen to result in lipotoxic cardiomyopathy ${ }^{22,23}$.

This present study investigates the mechanistic basis for TRF improvement in skeletal muscle by assessing transcriptomic data of DIO and GIO models under TRF, followed by providing in vivo functional validation to support muscle sequencing data. Our findings demonstrate that the expression levels of Gnmt, CG5955 and Sardh are commonly upregulated. Additionally, the expression level of Dgat2, a key gene required for triglyceride synthesis and a potential player in regulating insulin sensitivity, was commonly downregulated. In the DIO model, gene ontology (GO) analysis revealed significant upregulation in purine biosynthesis and de novo IMP (inosine monophosphate) production, in addition to immune related function, while $\mathrm{GIO}$ revealed upregulation in gene expression relating to glycolysis, glycogen metabolism, ETC and TCA connected by AMPK signaling ${ }^{34}$. Purine cycles play a role in energy balance, potentially regulating ATP levels required for optimal muscle performance ${ }^{24,25}$. Activation of AMPK protects against obesity through upregulation of downstream pathways which aid in metabolism and whole-body energy expenditure while improving glucose uptake and insulin sensitivity ${ }^{26,27}$. For muscle performance, validations of specific genes and pathways involved in each obesity model were completed to reveal mechanistic benefits of TRF. Additionally, biochemical measurements of ATP levels and phospho-AMPK levels were carried out under DIO and GIO to validate transcriptomic and genetic findings. Taken together, this study elucidates potential mechanisms behind TRF's protective properties against skeletal muscle dysfunction and metabolic impairment induced by obesity, which may pave the way for future TRF studies in muscle.

\section{Results}

Common differentially expressed genes (DEGs) identified under TRF in WT and both obesity models. To gain insight into the molecular mechanisms within skeletal muscle by which TRF protects against obesity-induced metabolic and functional changes, we measured the temporal transcriptomic profiles of indirect flight muscle (IFM) samples from WT, DIO and GIO flies under ALF and TRF conditions.

Feeding regimen and food type were assigned on day 4 such that flies under ALF had food access during the entire $24 \mathrm{~h}$, while flies under TRF had $12 \mathrm{~h}$ food access in daytime only (Fig. 1a). Wild-type Drosophila (Canton S) were subjected to either regular diet (WT control) or high-fat diet (DIO model). Sk2 mutants were fed with a regular diet (GIO model). IFMs were collected at week 3 from 15 female flies per cohort every $4 \mathrm{~h}$ over a 24-h period, and poly (A+) RNA-seq analysis was performed (Fig. 1b) (See Supplementary Table 1 for FPKM values).

We analyzed the transcriptomic data using two approaches. First, time-series samples were treated as replicates to identify DEGs between groups using DESeq2 ${ }^{28}$ (see Supplementary Table 2-4 for results of differential expression analysis in WT, DIO and GIO, respectively). As TRF is known to induce moderate changes in gene expression ${ }^{17}$, the differential gene expression threshold was set as fold change $\geq 1.25$ and a $p$ value $\leq 0.05$ in the analysis. Using these criteria, we identified 143,270 and 408 significantly upregulated genes, and 236, 636 and 579 significantly downregulated genes under TRF versus ALF in WT, DIO and GIO models respectively (Fig. 1c, d). Notably, fewer DEGs were detected in TRF versus ALF in WT than the obesity models, which implied that TRF may induce more regulation under metabolic stress. Principal component analysis (PCA) revealed gene expression differences between WT and obesity models, while the effect of feeding pattern (TRF versus ALF) was less clear (Supplementary Fig. 1a).

Second, we used empirical JTK_CYCLE ${ }^{29}$ to identify genes with a 24-h period cycling pattern of expression. Rhythmic genes in different groups were identified (Supplementary Fig. 1b-d) using criteria shown in the method section. Notably, more periodic transcripts were identified under TRF in WT and DIO, except GIO. Core clock genes Clk, Per and Tim, and Cyc, were examined as previous TRF studies 
have shown that TRF modulates circadian clock gene expression and rhythm in the liver of high-fat fed mice ${ }^{13}$. Interestingly, there were no significant expression differences between ALF and TRF for any core clock genes in WT and obesity models (Supplementary Fig. 2a-d). Clk and Tim exhibited periodic oscillations under both ALF and TRF in WT, DIO and GIO models (Supplementary Fig. 2e, g). Cyc did not show consistent rhythmicity (Supplementary Fig. 2f), which was aligned with the previous finding that Cyc mRNA does not cycle ${ }^{41}$. Per was unrhythmic at ALF but gained periodicity under TRF in DIO model (Supplementary Fig. 2h).

Given that TRF improved WT and different obesity models in a similar fashion ${ }^{11}$, we hypothesized that gene expression changes under TRF versus ALF which are shared among WT and obese flies could decipher the generic benefits of TRF. DEG analysis showed that 5 genes (CG6188, CG6385, CG5995, CG6806, and CG5896) were commonly upregulated and 3 genes (CG1942, CG7997, and CG13992) were commonly downregulated under TRF across WT, DIO and GIO models (Fig. 1C-g).

To investigate the roles of common upregulated genes in WT and obesity models, we examined their expression levels and functional annotations. 3 out of 5 common upregulated genes stood out as they were orthologous (CG6188/Gnmt, CG6385/Sardh, and CG5955/Tdh) to human genes and also shared a role in glycine utilization and production (Fig. 2a, Supplementary Fig. 3a). GNMT, glycine Nmethyltransferase, catalyzes the methylation of glycine by using S-adenosylmethionine (SAM) to form Nmethylglycine (sarcosine) with the concomitant production of S-adenosylhomocysteine (SAH). SARDH, sarcosine dehydrogenase, oxidatively demethylates sarcosine to glycine in mitochondria. Additionally, glycine can also be produced from CG5955 and glycine C-acetyltransferase (GCAT) ${ }^{30}$. However, CG5955 is a pseudogene and does not produce a functional protein in humans. The expression levels of Gnmt, Sardh and CG5955 were higher under TRF versus ALF in at least 4 out of 6 time points (Supplementary Fig. 3b).

Muscle-specific knockdown of Gnmt, Sardh and CG5955 leads to progressive skeletal muscle dysfunction, accumulation aberrant lipid accumulation and abolishes TRF-mediated benefits. To understand the roles of Gnmt, Sardh and CG5955 in skeletal muscle, we performed IFM-specific (Act88FGal4) RNAi KD of Gnmt, Sardh and CG5955 using three individual RNAi lines per gene with one initially tested siRNA validated in WT flies (Supplementary Fig. 3c). Muscle performance was measured using flight assay at 1-, 3- and 5-week-old female flies (Fig. 2c). Reduced flight performance was detected and progressed with aging during IFM-specific Gnmt suppression (with a 21\%, 44\%, and 54\% reduction at week 1,3 , and $5, p \leq 0.0001$ for all time points). Similarly, IFM-specific Sardh RNAi lines had significantly decreased flight ability (with a $25 \%, 36 \%$, and $47 \%$ reduction at week 1,3 , and $5, p \leq 0.0001$ for all time points). Finally, IFM-specific KD of CG5955 had strikingly reduced flight performance and was nearly flightless at week 5 (with an $84 \%, 88 \%$, and $89 \%$ reduction at week 1,3 , and $5, p \leq 0.0001$ for all time points). Similar reduction of flight ability was also observed in male flies at 1 week. (Supplementary Fig. 3d). The results indicate that the Gnmt, Sardh and CG5955 are essential for normal muscle performance. To test whether Gnmt, Sardh and CG5955 contribute to the improved muscle performance of TRF, we tested flight performance of WT flies with Gnmt, Sardh and CG5955 IFM-specific KD under ALF and TRF in 3-week-old flies. TRF failed to improve flight performance when compared to ALF flies (Fig. 2d), thus suggesting that Gnmt, Sardh and CG5955 are regulated under TRF to achieve beneficial effects observed in skeletal muscle performance.

Moreover, IFM-specific RNAi KD of Gnmt, Sardh and CG5955, resulted in the accumulation aberrant lipid accumulation, compared to age-match control, as we previously demonstrated in DIO and GIO models in the muscle tissue ${ }^{11}$. As shown in Fig. 2e, cryosections of IFMs from 3-week-old female flies upon IFM-specific KD of Sardh and CG5955 resulted in a significant upregulation of aberrant lipid accumulation (both size and density, see arrow) with Nile red staining. Furthermore, IFM-specific KD of Sardh and CG5955 resulted in an excess of actin-containing myofibrils (phalloidin staining, Fig. 2e, asterisks) compared to age-matched control myofibrils, which correlated with muscle dysfunction. IFMspecific $K D$ of Gnmt exhibited only moderate increase in lipid size however, the lipid density was significantly enhanced, compared to age-matched control tissue (Fig. 2 e-g). Overall, in addition to 
significant reduction in muscle performance, IFM-specific RNAi KD of Gnmt, Sardh and CG5955 resulted in a cytotoxic effect in the adipose tissue, potentially due to dysregulation of lipid homeostasis.

Muscle-specific knockdown of Dgat2 retains age-dependent decline of skeletal muscle function. CG1942 is orthologous to human Dgat2. DGAT2, known as one of the two acyl-CoA: diacylglycerol O-

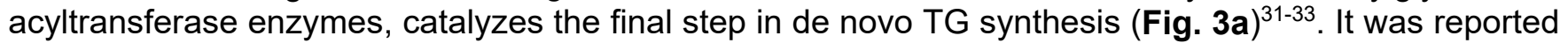
that the depletion of lipid droplet localized DGAT2 resulted in reduction of the large lipid droplet population ${ }^{34}$. The expression levels of Dgat2 were reduced under TRF versus ALF in at least four time points in WT, DIO and GIO models (Fig. 3b and Supplementary Fig. 4a). We also examined the expression of upstream enzymes associated with lipid droplet de novo TG synthesis. Interestingly, Gapt4 and Agpat3 also showed decreased but not significant expression levels under TRF versus ALF (Fig. 3a and Supplementary Fig. 4b). These data may explain our previous finding that TRF attenuated the degree of lipid infiltration and total lipid area in IFM of WT, DIO and GIO models ${ }^{11}$. To investigate the role of Dgat2 in skeletal muscle function, IFM-specific RNAi was used to suppress Dgat2 expression in WT flies. Muscle performance was measured at 1, 3, 5 and 7-week-old female flies. As expected, suppression of Dgat2 significantly improved flight ability with age when compared to control (with a 58\% and $133 \%$ increase at week 5 and 7, p $\leq 0.0001$ for all time points) (Fig. 3c). Cryosections of IFMs from 3-week-old female flies upon IFM-specific $K D$ of Dgat2 were examined at 3-week-old female flies (Fig. 3d). Significant reduction of lipid droplet area in IFM were observed in Dgat 2 KD flies compared to the control while no change on lipid density (Fig. 3e, f). Abdomen lipid staining was also performed to ensure the Nile red quantification of lipids represent the true physiology and no significant differences were observed (Supplementary Fig. 4c, d). Thus, lowering of de novo TG synthesis, especially the expression of Dgat2, may account for at least a part of the beneficial effect of TRF on muscle performance. CG7997 is another down regulated gene orthologous to human Gla (Supplementary Fig. 4f), galactosidase alpha, however, little is known about CG7997 in Drosophila. We tested muscle performance on WT flies with IFM-specific Gla RNAi, flight ability was also significantly improved as aging (with a $56 \%$ and $93 \%$ increase at week 5 and $7, p \leq 0.0001$ for all time points) (Supplementary Fig. $\mathbf{4 g}$ ).

TRF increases folate regulation and purine biosynthesis in the DIO model. As a limited number of common differentially expressed genes under TRF were observed between the two obesity models, one simple hypothesis was that TRF modulates each obesity type uniquely. Therefore, we interrogated gene expression changes by performing GO and Reactome pathway enrichment analysis for DIO and GIO DEGs separately.

Genes that were significantly upregulated under TRF versus ALF in DIO were enriched in immune response, proteolysis regulation, purine biosynthesis and amino acid metabolism (Fig. 4a, b), while downregulated genes were involved in neuronal signaling (Supplementary Fig. 5a, b). Interestingly, Gnmt, one of the commonly upregulated genes identified in our WT, DIO and GIO models, has been reported to play key role in promoting purine biosynthesis by regulating 5,10-meTHF, which is the entry point of folate into mitochondria ${ }^{35}$. Therefore, we interrogated the gene expression changes within the folate cycle and de novo purine biosynthesis (Fig. 4c). The de novo purine synthesis is a 10-reaction 6enzyme process that generates IMP from PRPP ${ }^{36}$. Strikingly, mRNA expression levels of all 6 genes (Prat2, Gart, Pfas, Paics, AdSL and Atic) encoding enzymes were significantly upregulated under TRF in DIO (Fig. 4c, d). Prps, located upstream of de novo IMP biosynthesis, as well as Nmdmc and Pug within the folate cycle, were significantly upregulated (Fig. 4c, d). As Prat2, the rate-limiting enzyme of de novo purine synthesis, has shown significant circadian rhythms in mice liver ${ }^{37}$, we examined whether the significantly upregulated genes identified from folate cycle and purine biosynthesis under TRF in DIO also have periodic expression. As shown in Fig. 4e, 8 genes were periodic under TRF; among them, 7 genes oscillated in both ALF and TRF with higher amplitudes under TRF, while Atic gained periodicity under TRF. All cycling genes reached the peak expression at the end of the eating period. Notably, increased but not significant expressions of these genes under TRF versus ALF were observed in WT and GIO, with a similar peak pattern shown in GIO (Supplementary Fig. 6a, b). 
To validate the roles of genes in folate cycle and purine synthesis in skeletal muscle, we performed IFM-specific $K D$ of $N m d m c$ and $A d S L$ using RNAi lines. Reduced flight performance was detected and was progressively worsened with age in IFM-specific Nmdmc suppression (with a $15 \%$ and $25 \%$ reduction at week 1 and 3). IFM-specific $K D$ of $A d S L$ led to complete flight loss at week 1 (Fig. 4f). To validate whether Gnmt and Nmdmc contribute to the improved muscle performance of TRF especially under DIO, we tested flight ability of DIO flies with Gnmt and Nmdmc KD under ALF and TRF in 3-weekold female flies. While TRF-mediated muscle improvements were observed in control flies, TRF failed to improve flight ability when compared to ALF in the suppression of Gnmt or Nmdmc (Supplementary Fig. 4c). As purine biosynthesis plays an important role in energy balance, ATP levels were measured from the thoraces of 3-week-old DIO female flies under ALF and TRF. The relative ATP levels increased by $20 \%$ under TRF compared to ALF in the DIO flies, while no changes were observed in WT flies (Supplementary Fig. 4d).

Folic acid, also known as vitamin B9, can be reduced to THF, and then enter the folate cycle in support of purine biosynthesis. Given the significant upregulation of Gnmt, Sardh, CG5955 and genes associated with purine synthesis under TRF in DIO, we tested the impact of folic acid supplementation on muscle function under a high-fat diet challenge. A titration of folic acid concentration was performed from $0.1 \mathrm{mM}$ to $16 \mathrm{mM}$. As expected, dose-dependent improvements were shown from $0.1 \mathrm{mM}$ to $4 \mathrm{mM}$ in flight and climbing performance (Supplementary Fig 6e), while higher concentrations of folic acid supplement led to adverse effects at $8 \mathrm{mM}$ and $16 \mathrm{mM}$. Therefore, $4 \mathrm{mM}$ was used in the following folic acid supplement assays as this concentration displayed the greatest beneficial effect. This improved muscle function observed in a high-fat diet supplemented with folic acid prompted us to test the effects of folic acid in WT and GIO conditions. However, no improvements in flight and climbing abilities were found in WT and GIO (Supplementary Fig 6f, g).

As folic acid supplement improves muscle performance in DIO flies, the effects of folic acid supplement in DIO were also examined in flies with IFM-specific KD of Gnmt and Nmdmc. Interestingly, significantly improved flight performance was observed in flies with IFM-specific $K D$ of $N m d m c$ under high-fat diet supplemented with folic acid, but not in flies with IFM-specific KD of Gnmt (Fig. $\mathbf{4 g}$ ). Significant increases of ATP levels were also detected in Nmdmc KD DIO flies supplemented with folic acid (Fig. 4h). All these results suggest that TRF increases purine synthesis within DIO and in turn ameliorates obesity related muscle phenotype with increases in ATP levels.

TRF upregulated genes linked with AMPK and downstream pathways in the GIO model. Based on the GO and Reactome analysis, genes that were significantly upregulated under TRF in GIO were enriched in four major groups: (1) Glycolysis; (2) Glycogen metabolism; (3) TCA cycle; (4) Mitochondrial electron transport chain (Fig. 5a, b). Additionally, GO and Reactome analyses revealed significant downregulation of many genes involved in cell division, DNA replication and DNA repair (Supplementary Fig. 5c, d). Interestingly, these four metabolic pathways enriched by upregulated genes under TRF can be connected by the activation of AMPK (Fig. 5c, d). Moreover, the mRNA levels of AMPK a subunit and genes associated with AMPK downstream pathway were significantly increased under TRF versus ALF in GIO, this trend was not seen in WT or DIO (Supplementary Fig. 7a, b). Previous studies have revealed rhythmicity of gene expression level for key enzymes of glycolysis in mice skeletal muscle ${ }^{38}$, and glycogen metabolism in rat liver ${ }^{39,40}$. Therefore, we examined whether the significantly upregulated genes identified from AMPK signaling downstream pathways under TRF in the GIO model also have periodic expression. As expected, most of genes encoding enzymes in glycolysis and glycogen metabolism showed periodicity under TRF (Fig. 5e), where Pgi, Pgk, and Eno gained rhythmicity under TRF versus ALF. Elevated amplitudes were detected among genes that oscillate in both ALF and TRF. On the other hand, most of the genes associated with TCA cycle and ETC didn't show periodicity, which were consistent with the previous observation that TCA cycle and ETC components exhibited circadian changes in protein but not mRNA levels ${ }^{41,42}$. Notably, expression levels of the upregulated genes associated with AMPK signaling downstream pathways were unchanged, if not decreased, under TRF in WT and DIO models (Supplementary Fig. 7b, c). Temporal profiles of other upregulated genes 
associated with glycolysis, glycogen metabolism, TCA cycle and ETC were visualized in heatmap, and consistently elevated expression appeared at the end of the eating period (Supplementary Fig. 7d).

To determine whether AMPK and its downstream pathways contribute to TRF-dependent skeletal protection, we first examined the AMPKa (the catalytic subunit of AMPK in Drosophila) protein status under ALF and TRF in WT, DIO and GIO. While AMPKa protein levels were unchanged across conditions (Fig. 5f, Supplementary Fig. 7e), reduced phosphorylated AMPKa (p-AMPKa) levels were observed under DIO-ALF and significantly under GIO-ALF compared to WT-ALF (Fig. 5f, g). Notably, a consistent increase of p-AMPK levels was present under TRF with most increase in GIO compared to ALF (Fig. 5f, g), which implies the potential upregulation of TRF-dependent AMPK activation especially in the GIO model. We tested flies with IFM-specific $K D$ of $A m p k \alpha$ and selected enzymes associated with glycolysis, glycogen metabolism, TCA and ETC. KD of Ampka, GlyP, GlyS, Pfk, Ald1, mAcon1, sicily, SdhD and CG15738 led to significant reduction of flight performance in 1- and 3-week-old flies (Fig. 5h). To further validate the GIO-specific roles of AMPK downstream pathways, we tested the flight performance of flies with GlyS and mAcon1 IFM-specific knockdown accompanied with Sk2 RNAi. While mAcon1 RNAi itself led to flight performance reduction, $m A$ con 1 and $S k 2$ double $K D$ caused further reduction in flight index (Supplementary Fig. 7f). A similar trend was also observed in GlyS KD. As AMPK is known as an energy sensor, ATP levels were also measured under ALF and TRF in 3-week-old GIO female flies. The relative ATP levels were reduced by $32 \%$ in GIO ALF compared to WT ALF, and as expected, TRF rescued the relative ATP levels by $20 \%$ compared to ALF in the GIO condition (Supplementary Fig. 6d). Taken together, our results suggested that TRF activates AMPK signaling and its downstream pathways through increased gene expression in a GIO specific manner. Consequently, this change in gene expression leads to increased ATP levels and in turn ameliorates obesity muscle related phenotypes.

\section{Discussion}

Skeletal muscle plays a primary role for energy and protein metabolism, glucose uptake and storage. TRF confers protection against obesity by maintaining metabolic homeostasis in peripheral tissues such as heart and skeletal muscle ${ }^{11,43}$. Underlying pathways and mechanisms involved in TRF protection against diet and genetic induced obesity in muscle remain unexplored. The first objective of our study sought to identify common DEGs in IFM under TRF comparatively to ALF in WT and obesity models and secondly identify DEGs that are distinct in DIO TRF and GIO TRF. (Fig. 1c). Genes orthologous to humans were selected for further analysis in both up and downregulated genes. TRF upregulated expression of Gnmt, Sardh, and CG5955, all genes which may play a role in regulating Sadenosylmethionine (SAM) levels, a universal methyl donor shown to be increased in aging flies ${ }^{9,44}$. TRF downregulated Dgat2 a key enzyme related to triglyceride synthesis ${ }^{45}$. Furthermore, TRF activated metabolic pathways specific to obesity types: purine synthesis in DIO and AMPK signaling and related downstream pathways in GIO (Figs 4a-f and 5a-f).

SAM can play a therapeutic role in liver disease and yet also displays pathogenicity leading towards simple steatosis and non-alcoholic steatohepatitis (NASH) ${ }^{46-48}$. Additionally, SAM levels have been observed to correlate with age in flies ${ }^{44}$. Increased levels of SAM can disrupt the sadenosylmethionine sensor upstream of mTORC1 (SAMTOR) leading to mTORC1 activation known to induce insulin resistance in obesity ${ }^{49-51}$. Such studies underscore the role of SAM in controlling metabolism; how SAM may negatively impact muscle and metabolism remains to be explored, however. In our study, we found that TRF increased the expressions of Gnmt, a chief regulator of SAM in addition to Sardh and CG5955, glycine producers which may assist SAM catabolism ${ }^{9,44}$ (Fig. 2a). Upon further inspection, IFM-specific $K D$ of these genes displayed significant reduction in flight index shown in Fig. 2c. Interestingly, imposing TRF in these $K D$ models showed no significant improvement on flight index signifying the importance of these three genes potentially required for TRF mediated muscle improvement (Fig. 2d). From this, successful SAM regulation in obese conditions may potentially be a 
critical factor in TRF's ability to help regulate metabolic homeostasis and skeletal muscle function. A recent clinical study observed a correlative increase in SAM levels in older adults with increased fat mass and truncal adiposity suggesting a role in obesity. Additionally, a related study observed that SAM was increased in overfed humans ${ }^{8,52}$ further supporting the findings. Another recent study also revealed that upon a high fat diet challenge in mice, GNMT was downregulated ${ }^{53}$. There is currently little known regarding the role of GNMT in skeletal muscle, however both mRNA and protein levels have been observed in the skeletal soleus in mice ${ }^{54}$. Additionally, Sardh is abundantly expressed in the muscle of humans according to the human protein atlas. CG5955 is a pseudogene expressed in mammals but does not lead to a functional protein lacking most of its C-terminus along with parts needed for NAD+ binding motif; however, functional CG5955 is present in models such as mice ${ }^{55}$. Our results are first to demonstrate that Gnmt, Sardh and CG5955 are essential to TRF's ability to promote skeletal muscle function demonstrated by lack of TRF improvement in $K D$ of these genes in Drosophila (Fig. 2d). Dgat2 downregulation is also metabolically noteworthy as lipid accumulation and insulin resistance observed in mice overexpressing Dgat2 was observed in glycolytic type 2 muscle ${ }^{56}$. Interestingly, the flight index of Dgat2 KD showed improvement in performance highlighting the importance of its regulation in muscle (Fig. 3c). From this, TRF irrespective of obesity background may help maintain skeletal function through SAM reduction via Gnmt, Sardh and CG5955 and also through reduction of Dgat2.

Transcriptome analysis using GO and Reactome showed that TRF mostly upregulated purine related cycles and immune related response in the DIO model (Fig 4a, b). The purine cycle helps balance energy requirements potentially needed for muscular contraction through serveral ways including replenishment of the citric acid cycle intermediate of fumarate and increasing flux for adenylate kinase ${ }^{57}$. A key gene for both cycles, adenylosuccinate lyase (AdsL) was found to be significantly upregulated along with most upstream enzymes (Fig. 4c, Supplementary Table 5) and interestingly KD of the AdsL gene showed complete impairment of flight index (Fig 4f). Additionally, a recent human study comparing 11 human obese individuals under TRF (8 h eating window) and extending feeding (EXF; $15 \mathrm{~h}$ window) showed purine de novo IMP genes upregulated as well ${ }^{14}$ (Supplementary Fig. 8a, b, Supplementary Table 7). These results may indicate the translatable significance of the purine cycle in humans, though the cause of obesity from this study was undisclosed and was performed in a short-term TRF period with a $15 \mathrm{~h}$ eating window period instead of $24 \mathrm{~h}$. The importance of ADSL plays a role in ATP generation and a new role of ADSL has been recently uncovered as an insulin secretagogue leading to insulin release and glucose uptake ${ }^{58}$. In addition to regulation of Dgat2, AdSL may also be key in TRF related mechanisms in combating insulin resistance in DIO. Interestingly, a study showed that AdSL deficiency during moderate aerobic stimulation was associated with muscle dysfunction ${ }^{25}$ implying its importance in TRF mediated regulation in muscle function as well. This in turn gives reason to suggest that TRF improves glucose metabolism and skeletal muscle function in the DIO model through the purine related cycle and through upregulation of AdSL. Also, production of 10 formyl tetrahydrofolate (10 formylTHF) is key to purine cycle and serum folate levels observed to be negatively correlated with insulin resistance and obesity ${ }^{59}$. Pug and Nmdmc help produce 10 formylTHF and were found to be significantly upregulated (Fig 4d, Table S6) supporting the case of purine cycle involvement in TRF. Folate is a key component for purine synthesis and maintaining folate levels are crucial to DNA synthesis, immune related response, and production of key biomolecules and consequently, deficiency leads to cardiovascular disease, muscle weakness, and difficulty in walking ${ }^{60-62}$. KD of Nmdmc displayed significant reduction in flight index exemplifying its importance in muscle function (Fig. 4f) while folate supplementation demonstrated flight improvement and increase in relative ATP levels (Fig $\mathbf{4 g}, \mathbf{h}$ ). This is to be expected if folate levels are indeed crucial for purine related cycles and if in fact the purine cycle contributes to muscle performance. Interestingly, Gnmt expression may also promote nucleotide biosynthesis through its aid in production of 5, 10-methylene tetrahydrofolate with Sardh, which is the entry point of folate into mitochondria ${ }^{63}$. Additionally, GNMT helps purine expression through its translocation to the nucleus in folate depleted conditions potentially linking its contribution to DIO conditions in regard to the activation of purine related pathway ${ }^{9,63,64}$. 
In the GIO model, GO and Reactome pathway results yielded enrichment in tricarboxylic acid cycle, glycogen metabolism, glycolysis, and mitochondrial electron transport chain (ETC) (Fig 5a, b, Supplementary Table 7). These pathways are connected through AMPK signaling, also shown to be significantly upregulated in TRF (Fig. 4c) ${ }^{65,66}$. AMPKa is the catalytic domain homolog seen in Drosophila and acts as an energy sensor able to sense high ratios of AMP/ATP and help regulate lipid metabolism 67 found to be significantly increased in expression (Supplementary Table 7). AMPK is linked to catabolism and provides energy for muscle contraction through ATP production in glycolysis and ETC, mediating glucose uptake in tissues such as muscle and mediating fatty acid oxidation ${ }^{65,68}$. Results showed consistent increase in ETC genes with only 1 gene showing unchanged expression out of 57 found and TCA demonstrated significant increases in key enzymes (aconitase, isocitrate dehydrogenase, $\alpha$-keto glutarate dehydrogenase). p-AMPKa was measured and as expected was found to be increased in GIO (Fig 5f). Furthermore, the flight index of $K D$ genes in each pathway demonstrated significant impairment (Fig 5h) and further decrease in flight ability was seen in a genetic induced obesity background (Supplementary Fig. 7g). Comparing our data to a recent human study with 11 human obese individuals, human transcriptome ${ }^{14}$ did not mirror the changes found in the GIO model (Supplementary Table S8). This may be due to participants coming from a DIO background rather than GIO. Interestingly, our previous study demonstrated that ETC was downregulated in the aging heart of WT under TRF flies and their cardiac-specific $K D$ consequently led to improved cardiac performance during aging ${ }^{17}$. It is to be noted however, that this previous study was performed in old wild-type heart tissue rather than middle age GIO skeletal muscle therefore metabolic demands led to their expression. Downregulation of ETC may suggest that TRF mediates cardiac function through reduction of reactive oxygen species which may not be necessary in skeletal muscle as skeletal muscle fibers have a much higher oxidative capacity seen in healthy humans ${ }^{69,70}$. Of note, GNMT's regulation of SAM level plays a role in AMPK activation indirectly as SAM's methylation of protein phosphatase 2A (PP2A) inhibits AMPK activation and GNMT reduction of SAM can therefore lead to AMPK activation ${ }^{71}$ (Fig 5c). This may suggest that GNMT plays a role in activating AMPK by inhibiting SAM related inhibition. Additionally, Forkhead Box O (FoxO) was significantly increased in expression with modest increase in unc-51 like autophagy activating kinase 1 (Ulk1) (Supplementary Table 7) potentially suggesting increased control of skeletal muscle mass ${ }^{70}$ through autophagy ${ }^{72}$. Results suggest that AMPK signaling is responsible for TRF mediated benefits found in skeletal muscle in the GIO model. Activation of AMPK reduces markers associated with skeletal muscle fragility ${ }^{73}$ and is additionally able to improve the oxidative capacity of muscle fibers through biogenesis of mitochondria ${ }^{74,75}$ which is seemingly in alignment with the results.

In summary, TRF previously exhibited improvement in metabolic and skeletal muscle function ${ }^{17}$ seen in Drosophila and this current study provides an innovative and mechanistic basis for this finding. Through analysis of muscle transcriptomic data from Drosophila and their genetic validation and biochemical analysis, we discovered that SAM regulation through Gnmt, Sardh and CG5955 in addition to triglyceride synthesis via Dgat2 were modulated commonly across all conditions under TRF. Common modulation of regulated genes has demonstrated their impactful roles in metabolic homeostasis and were additionally found in this study to greatly impact muscle performance. Additionally, common modulated genes may play a role in mediating DIO and GIO exclusive pathways in TRF. GNMT's translocation to the nucleus has been suggested to lead to purine activation and additionally SARDH also leads to folate entry points necessary for purine cycle. Secondly, reduction of SAM levels by GNMT indirectly activates AMPK by reduction of PP2A. Pathways discovered to be related to TRF mechanism were found to be purine cycles in DIO and AMPK related signaling and downstream pathways predominantly in GIO. This hypothesis is supported by measurement of ATP levels and p-AMPKa levels and validated by flight performance in $K D$ of key genes in each pathway. The results highlight the metabolic and functional importance of purine biosynthesis and AMPK downstream signaling within the skeletal muscle in different obese models under TRF and the potential impact of SAM and triglyceride synthesis regulation in the TRF mechanism (Fig. 6). 


\section{Methods}

Drosophila models, diets, and feeding fasting regimens. Canton-S and Sphingosine kinase 2 (Sk2, $\mathrm{BL}$ \#14133) were obtained from Bloomington (BL) Drosophila Stock Center. Sphingosine kinase 2 is the Drosophila homolog of human Sphingosine kinase 1. Sk2 mutants show a hallmark accumulation of ceramide, which has been implicated as a contributor to obesity ${ }^{22}$. Standard regular diet (RD): agar $11 \mathrm{~g} / \mathrm{L}$, active dry yeast $30 \mathrm{~g} / \mathrm{L}$, yellow cornmeal $55 \mathrm{~g} / \mathrm{L}$, molasses $72 \mathrm{~mL} / \mathrm{L}, 10 \%$ nipagen $8 \mathrm{~mL} / \mathrm{L}$, propionic acid $6 \mathrm{~mL} / \mathrm{L}$. High-fat-diet (HFD): standard diet supplemented $+5 \%$ coconut oil ${ }^{17}$. For the WT control model, CS flies were fed with RD. To model diet-induced obesity, CS flies were fed with HFD. To test the impact of folic acid, $4 \mathrm{mM}$ of folic acid was added either to high-fat diet or standard diet. $4 \mathrm{mM}$ concentration of folic acid was determined for use through a titration of different folate concentrations $(0.1 \mathrm{mM}, 1 \mathrm{mM}, 4 \mathrm{mM}, 8 \mathrm{mM}$ and $16 \mathrm{mM})$ where $4 \mathrm{mM}$ showed the most beneficial impact in muscle performance (Supplementary Fig. 6d). To model genetic-induced obesity, Sk2 flies were fed with RD. Male and female adult flies were collected upon eclosion, and maintained on a standard diet in groups of 20-25 for 3 days. Vials were assigned a diet and feeding regimen on the fourth day. Flies were transferred onto fresh media every day. ALF and TRF flies were subjected to vials with assigned diet at zeitgeber time zero (ZT 0) 9AM (lights on), and were switched to either a new food media vial (for ALF) or a $1.1 \%$ agar vial (for TRF) at 9 PM (lights off). All flies were kept at $22{ }^{\circ} \mathrm{C}, 50 \%$ humidity in a $12 \mathrm{~h}$ light/12 h dark (LD) cycle ${ }^{11}$.

To evaluate skeletal muscle-specific function of the identified genes, the following UAS-RNAi transgenic stocks were obtained from Bloomington Drosophila Stock Center (BDSC) and Vienna Drosophila Resource Center (VDRC): Gnmt-RNAi (BDSC: 42637; VDRC: 110623; VDRC: 25983), Sardh-RNAi (BDSC: 51883; VDRC: 108873, VDRC: 27601), CG5955-RNAi (BDSC: 64566, VDRC: 102443; VDRC:15838), Dgat2-RNAi (VDRC: 107788), Nmdmc-RNAi (BDSC: 62268), Ampka-RNAi (BDSC: 57785), GlyS-RNAi (BDSC: 50956), GlyP-RNAi (VDRC: 27928), Pfk-RNAi (BDSC: 34336), Ald1-RNAi (BDSC: 26301; BDSC: 65884), Nc73EF-RNAi (BDSC: 33686), mAcon1-RNAi (BDSC: 34028; BDSC: 24756), SdhD-RNAi (BDSC: 65040), CG15738-RNAi (BDSC: 55442), Control lines (BDSC: 36303, BDSC: 36304). Data from RNAi lines were combined if more than one RNAi line was used, unless otherwise indicated. Actin88F-Gal4 line was obtained from the laboratory of Richard Cripps.

RNA-Seq expression profiling. After 3 weeks of age on the assigned diet and feeding regimen, thoraces were collected from 5 flies every $4 \mathrm{hr}$ over $24 \mathrm{~h}$. Samples were flash frozen, ground to fine powder in liquid nitrogen. Tissues were homogenized using a Polytron homogenizer (Kinematica) and total RNA were prepared using the RNeasy kit (QIAGEN). Libraries were prepared according to Illumina protocols for single-end 50 bp mRNA sequencing and sequenced on an Illumina GA-II machine. Reads were aligned to the Drosophila melanogaster genome (BDGP r5) using STAR (v2.3.0e). Only reads aligning uniquely to the genome were considered. Normalized gene expression values (FPKM) were found by counting alignments overlapping with exons from RefSeq gene models using HOMER. WT-TRF ZT11 and GIO-ALF ZT23 were detected as outliers, therefore, data from these two time points were removed during analysis. Average values from adjacent time points were used for plotting.

Analysis of differential expression was carried out using DESeq $2^{28}$, with designs that accounted for models (WT, DIO and GIO), feeding group (ALF and TRF) and time of sample collection (subjective fed=ZT7-ZT12; subjective fast= ZT15-ZT24). Statistical significance was assessed using a negative binomial Wald test. The threshold of differential gene expression was set as fold change $\geq 1.25$ and a $p$ value $\leq 0.05$. For visualizations, a regularized logarithm transformation ${ }^{28}$ was applied to the gene-level read counts. Gene expression heatmaps were generated from these transformed values following mean-centering and hierarchical clustering. Principal component analysis was performed using the 500 
genes with highest variance across all samples. Gene Ontology Biological Process analysis and Kyoto Encyclopedia of Genes and Genomes (KEGG) pathway enrichment analysis were performed by the Database for Annotation, Visualization and Integrated Discovery (DAVID) v6.8. The GO terms were manually curated so that there were no more than three genes that were shared between any two GO terms.

To analyze the temporal dynamic of gene expression, we used empirical JTK_CYCLE with asymmetry search $^{40}$. Periodic patterns were determined using duplicated data i.e. two cycles were artificially created from the one actual cycle of experimental data ${ }^{11}$. Transcripts with a maximum/minimum foldchange $\geq 1.3$ and a Benjamini-Hochberg corrected $p$ value $\leq 0.05$ were considered periodic.

Flight muscle performance. To evaluate the roles of identified genes in muscle performance, flight test was conducted using flies with IFM-specific $K D$ of selected genes. Briefly, this methodology ${ }^{11,20,75}$ involves release of control and experimental adult flies into the center of a Plexiglas box with a light source positioned at the top (20-400 flies were tested for each gene using females). Based upon each animal's ability to fly up [6.0], horizontally [4.0], down [2.0], or not at all [0.0], we assign flight indexes (FI) for different ages and genotypes of flies. In all figures, the Fls were calculated for flies of different ages, genotypes, and food conditions.

Negative geotaxis. Flies were transferred to a new vial (10 flies per experiment) and allowed to rest for $2 \mathrm{~min}$ to acclimatize. The vial was then sharply tapped three times to stimulate a negative geotaxis response. Climbing ability of the flies was video-recorded and saved for data analysis. At $10 \mathrm{~s}$ intervals, the fraction of flies that climbed to the $7 \mathrm{~cm}$ mark was calculated.

Cytological analysis of muscle and abdomen tissues. As previously established ${ }^{11}$ for cytological analysis, sample were prepared and fixed in 4\% PFA, washed with PBS and then aligned longitudinally (thoraces) in a cryomold filled with Tissue-Tek OCT (Sakura), and then flash frozen in dry ice. $30-\mu \mathrm{m}$ thickness cryosectioned samples were washed and stained with 1X fluorescene dye 488-I with Phaloidin conjugate for the structural aberrations, and Nile red (at $1 \mu \mathrm{g} / \mathrm{mL})$ in $1 X$ PBS for the lipid staining. Fluorescence images were taken using Olympus BX-63 microscope coupled with cell-tracking software and quantification of lipid droplets (size and density) was performed using Python scripting language (CITE) and consisted of images simple binary and Otsu thresholding and watershed segmentation.

Western blot analysis. Five thoraces were added to $75 \mu$ of lysis buffer $(62 \mathrm{mM}$ Tris $\mathrm{pH} 7.5,0.1 \%$ SDS with protease and phosphatase inhibitors). The samples were boiled for $5 \mathrm{~min}$ at $95^{\circ} \mathrm{C}$ and centrifuges to collect the supernatant. Sample loading buffer was added, and the samples were then electrophoresed on 10\% Mini-PROTEAN TGX precast protein gels (Bio Rad). The protein bands were transferred to the nitrocellulose membrane (Bio Rad) and incubated with AMPK-alpha antibody (Cat.: ab80039, 1:3000, Abcam), Phospho-AMPK-alpha (Thr172) antibody (Cat.: 2535S, 1:1000, Cell Signaling), alpha Tubulin antibody (Cat.: ab80039, 1:3000, Abcam). For the secondary antibody, horseradish peroxidase-conjugated Goat anti-rabbit IgG (ab6721, Abcam) and Rabbit anti-mouse IgG (ab6728, Abcam) were used at 1:10000 dilution. Clarity Max Western ECL substrate was used to detect the signal, and the corresponding band was quantified using ImageJ software. Western blot was performed in triplicates for each genotype under ALF and TRF.

Real-time quantitative PCR. Dissected IFM from 1-week-old flies was placed in the RNA lysis buffer, and flash frozen. RNA was extracted using Zymo Research Quick-RNA Microprep Kit with on column 
DNase I digestion. Quantitative PCR was performed using SsoAdvanced Universal SYBR Green supermix (Bio Rad) in a BIO-RAD CFX Opus Real-Time PCR System. Expression was normalized with 60S ribosomal protein (RPL11). Primers for qPCR are listed below: Gnmt-F:

ACACGGCGGACATCAAG; Gnmt-R: TCGATCAGGTGAATGTAGAAG; Sardh-F:

CTGTCACACACTGTATCACCTG; Sardh-R: CGACGTGAATTAGCCAGCAG; CG5955-F: AGCCCAGTCAGTCGGTACT; CG5955-R: CAGTCAATGCGATGGTCCACA; Tdh-F: AGCCCAGTCAGTCGGTACT; Tdh-R: CAGTCAATGCGATGGTCCACA; Dgat2-F: TGTCCAAGTTGTTGGTGCTC; Dgat2-R: GGCACTCTTCGAATTCTCCA; Nmdmc-F: GCACCCAACTAGCACACGA; Nmdmc-R: CGACCATCTTGTTAGCCACATAC; Rpl11-F: CGATCTGGGCATCAAGTACGA; Rpl11-R: TTGCGCTTCCTGTGGTTCAC. Results are presented as $2^{-\Delta \Delta C t}$ values normalized to the expression of Rpl11 and control samples. All reactions were performed in triplicate. The means and standard deviations were calculated in GraphPad Prism 8 software.

ATP Quantification. ATP relative quantification levels were obtained using 10 3-week-old fly thoraces with a luciferase based ATP kit (Thermo Fisher). Briefly, fly thoraces were homogenized using a homogenization buffer (6 M guanidine HCL, $100 \mathrm{mM}$ Tris ( $\mathrm{pH} 7.8$ ), $4 \mathrm{mM}$ EDTA) and a motorized pestle. A small volume of the homogenate was used for detecting protein levels using a bradford assay (Bio-Rad). The remaining homogenate was lysed via boiling for 5 minutes and was centrifuged at maximum speed for 3 minutes in $4^{\circ} \mathrm{C}$. Dilution of the luciferin and luciferase were made per the instruction of the ATP kit and ATP dependent luminescence was measured at $560 \mathrm{nM}$. ATP measurements were taken from 3 samples per condition and were averaged after a total of 3 sequential readings which were normalized according to protein levels; both protein and ATP were measured using the EPOCH Biotek plate reader.

Statistical Analysis. Significance in climbing ability and flight performance was determined using oneway ANOVA with post-hoc Sidak's method for multiple comparisons. Bar graphs show mean \pm SEM. Lipid droplet area and density differences were performed by two-tailed unpaired t-test. Bar graphs show mean $\pm S D$. For western blot analysis, differences between samples were determined using twotailed paired t-test. Bar graphs show mean \pm SEM. Relative ATP quantifications analyses were made using a one-way ANOVA with Dunnett's post-hoc method for multiple comparisons. Bar graphs show mean \pm SEM. Significance was presented as ${ }^{n s} p>0.05,{ }^{*} p \leq 0.05,{ }^{* *} p<0.01,{ }^{* * *} p<0.001$. All statistical analysis were performed with GraphPad Prism 9. 


\section{Figure 1}

a
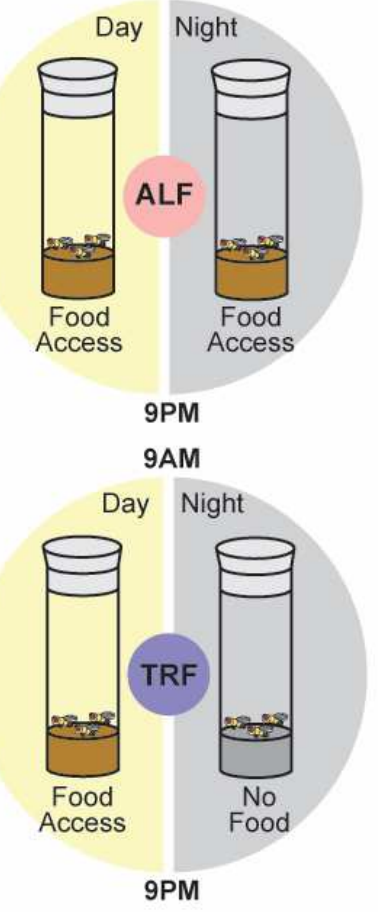

C Up Genes under TRF

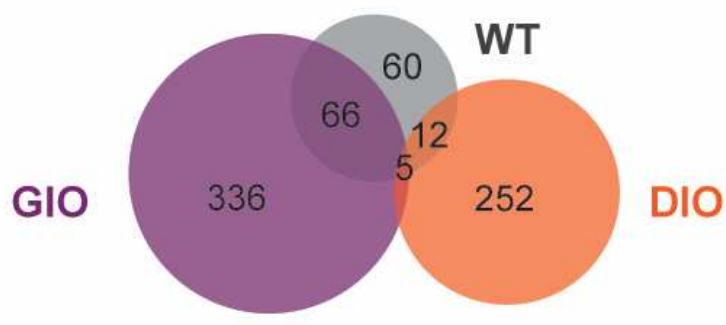

e

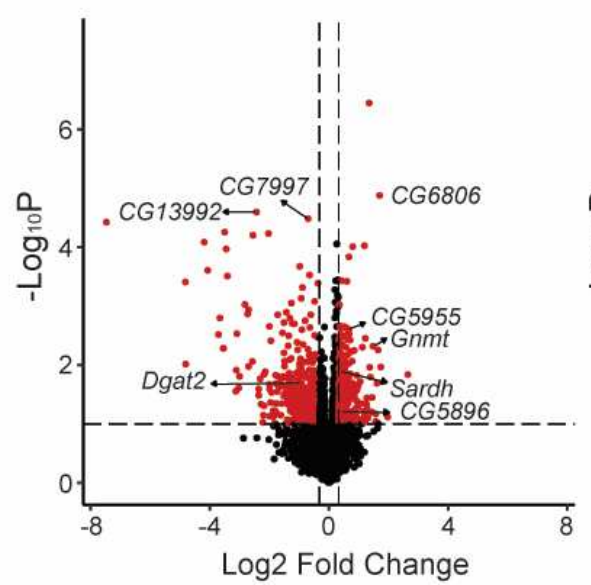

b
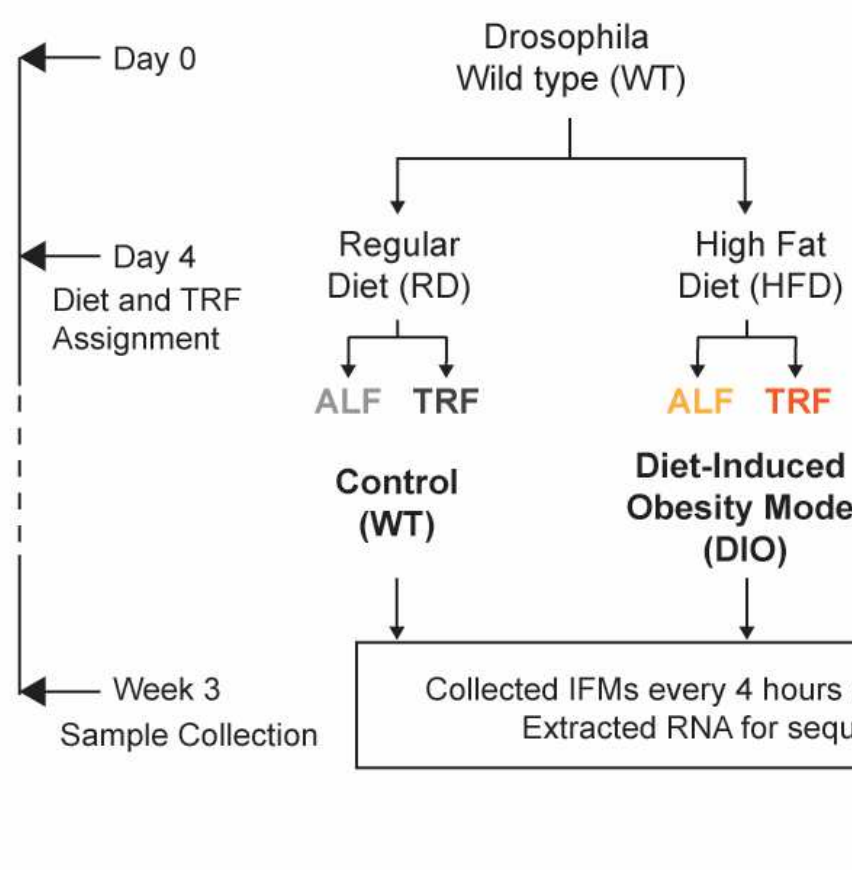

Control

(WT)

Diet-Induced Obesity Model

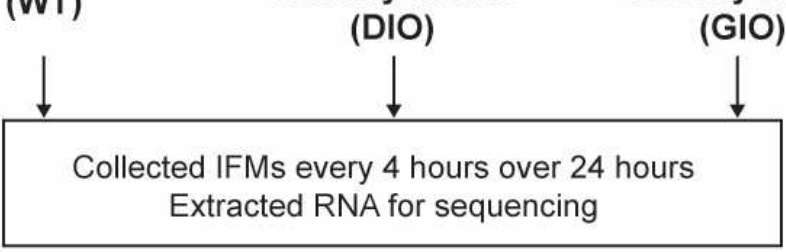

d Down Genes under TRF

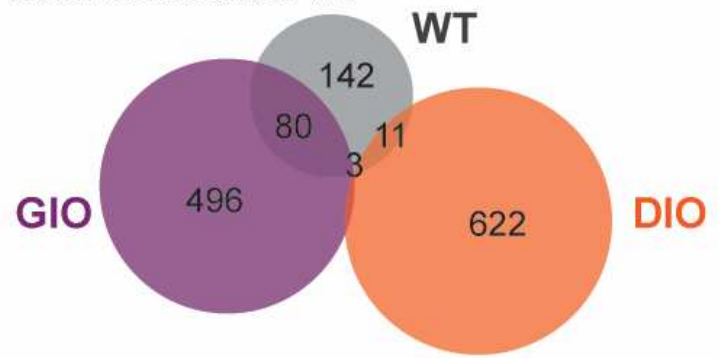

Drosophila

Genetically

Obese (Sk2)

Regular

Diet (RD)

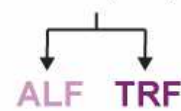

Genetic-Induced

Obesity Model

(GIO)

g
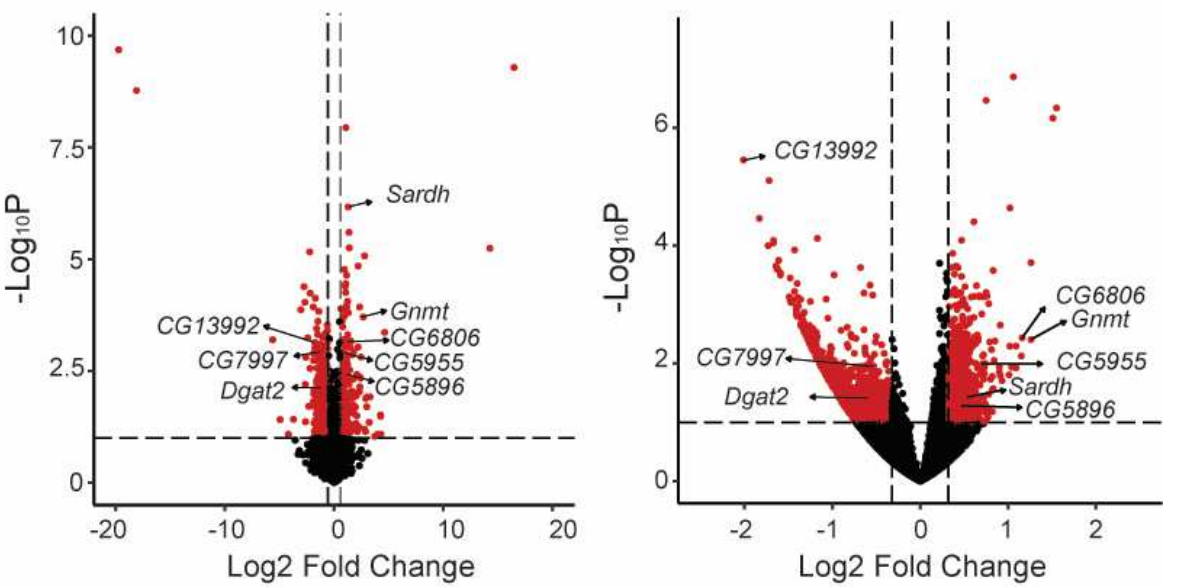

Figure Legends Figure 1: Common differentially expressed genes identified under TRF versus ALF in WT and obesity models. 
(a) Schematic depicting the timing of food access in ALF (ad libitum feeding) and TRF (time-restricted feeding). In ALF, flies have unrestricted access to food. In TRF, flies could consume food only during the $12 \mathrm{~h}$ of daytime.

(b) Flow chart depicting the experimental set up. Wild type and genetic obese mutant Sk2 flies were separated into respective food conditions regular diet (RD) or high fat diet (HFD) to establish WT, GIO and DIO models. Fly models were further separated into either ALF or TRF. Thoraces were collected at 3 weeks of age every 4 hours totaling to 6 time points for RNA sequencing.

(c) Venn diagram of significantly upregulated genes performed using DeSeq2 ( $p$ value $\leq 0.05$, Fold change $\geq 1.25$ ) under TRF in WT, DIO and GIO models.

(d) Venn diagram of significantly downregulated genes using DeSeq2 ( $p$ value $\leq 0.05$, Fold change $\geq$ 1.25) under TRF in WT, DIO and GIO models.

(e-g) Volcano plots showing expression profiles of common DEGs under TRF versus ALF in WT (e), DIO (f) and GIO (g) models. Red dots represent differentially expressed genes under TRF ( $p$ value $\leq 0.05$, Fold change $\geq 1.25$ ). 


\section{Figure 2}

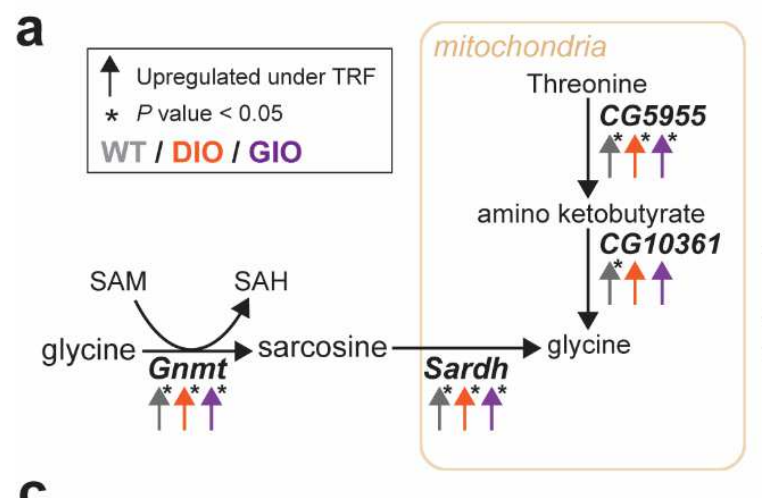

C
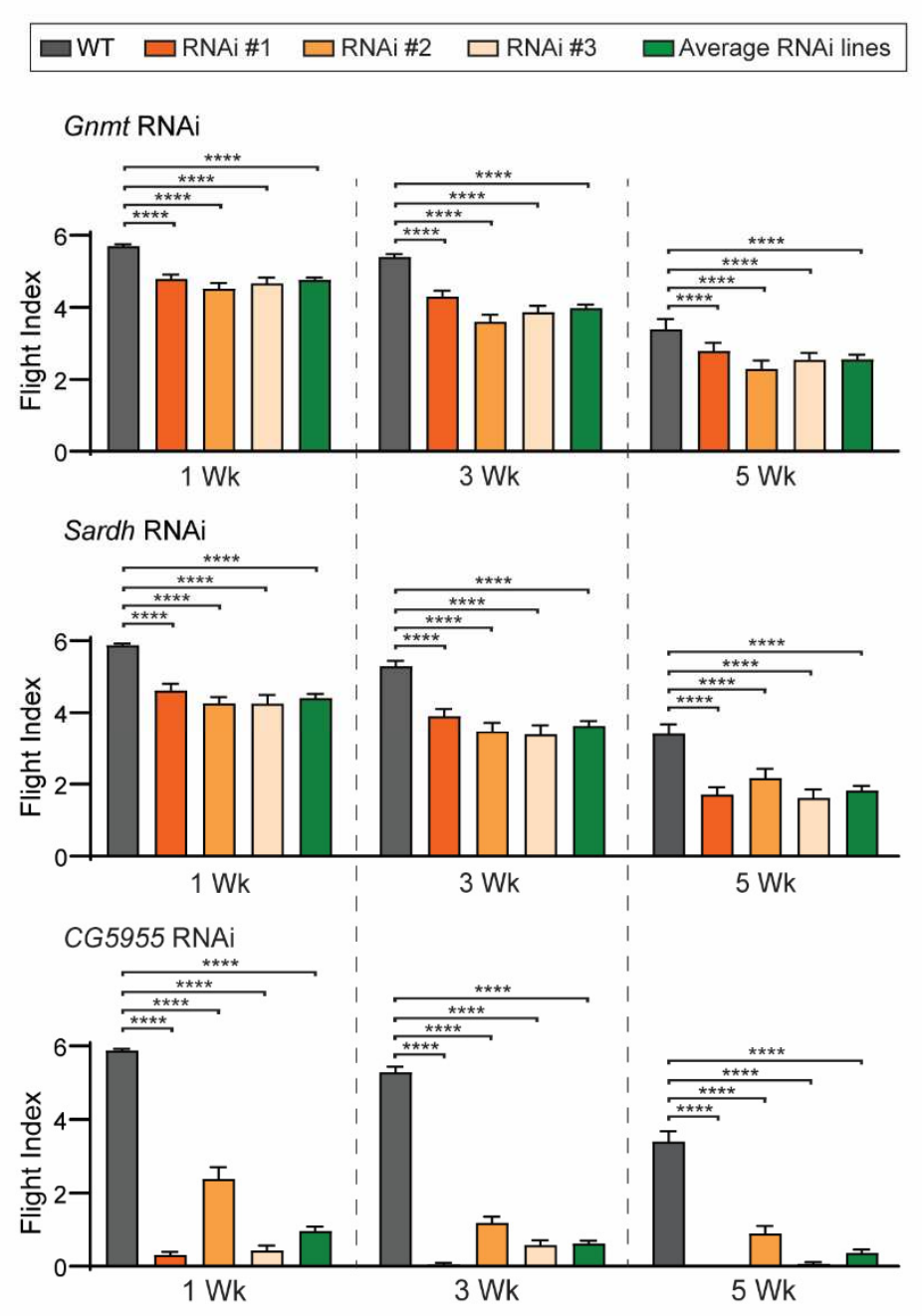

b
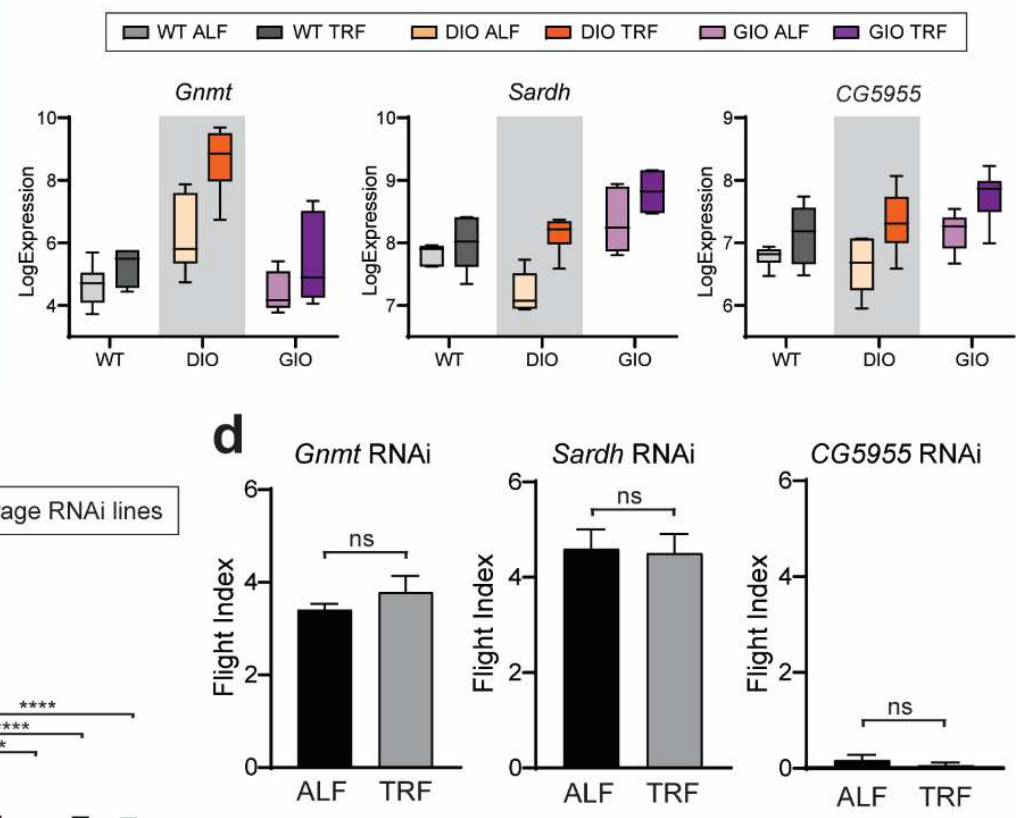

e

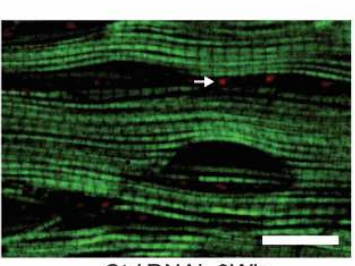

Ctrl RNAi, 3Wk

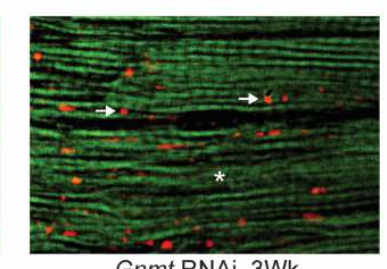

Gnmt RNAi, 3Wk
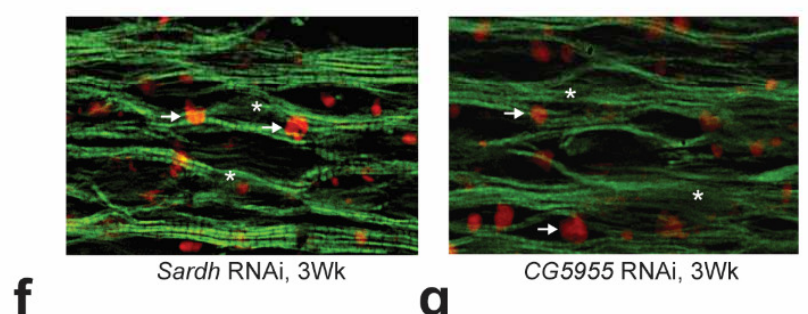

g

CG5955 RNAi, 3Wk
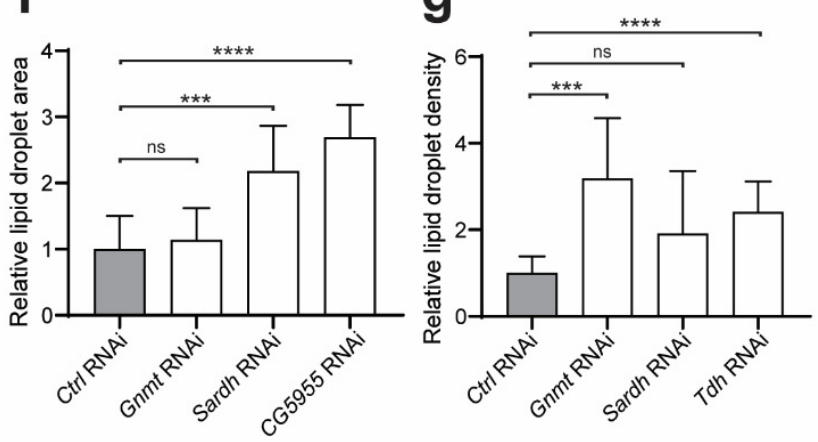

Figure 2: Gene expression and functional validation of Gnmt, Sardh and CG5955 in skeletal muscle in WT and obesity models.

(a) Schematic representation of Gnmt, Sardh and CG5955 connection with glycine utilization and production with metabolite intermediates and important genes (italic bold) encoding enzymes. Up arrows 
indicate upregulation of gene expression (fold change $\geq 1.25$ ) and color indicates condition (grey WT, Orange $\mathrm{DIO}$, purple $\mathrm{GIO}$ ). Asterisk indicates $p$ value $\leq 0.05$ and fold change $\geq 1.25$.

(b) Expression level (normalized read count, log2) of gene Gnmt, Sardh and CG5955 under ALF and TRF in WT, DIO, GIO models.

(c) Flight performance of WT female flies with Gnmt, Sardh or CG5955 IFM-specific KD at 1 week, 3 week and 5 weeks of age. Three independent RNAi lines per gene were tested and plotted individually. $\mathrm{N} \#=100-170$ females for each independent fly line per time point ( $\mathrm{N \#}=335-528$ combined).

(d) Flight performance of $K D$ in common upregulated genes at 3 weeks of age unchanged in TRF condition. As the three independent lines showed similar results in (c), one fly line from an independent batch was used and $\mathrm{N \#} \mathrm{=} \mathrm{50-60} \mathrm{females} \mathrm{for} \mathrm{each} \mathrm{condition.} \mathrm{Bar} \mathrm{graphs} \mathrm{represent} \mathrm{mean} \mathrm{and} \mathrm{SEM,} \mathrm{with}$ significance presented as ${ }^{\mathrm{ns}} p>0.05,{ }^{*} p \leq 0.05,{ }^{* *} p<0.01,{ }^{* * *} p<0.001$.

(e) Fluorescence images of the IFM from 3-week-old females of control and IFM-specific KD of Gnmt, Sardh and CG5955 flies upon probing with phalloidin (green) and Nile Red (red puncta). More accumulation of lipid (arrows) and actin-containing myofibrillar disorganization was detected in IFM of $K D$ of Gnmt, Sardh and CG5955 (asterisks), compared with age-matched control. Scale bar is $20 \mu \mathrm{m}$.

(f-g) Intramuscular lipid quantification (size (f) and density (g)) showed significant increase upon IFMspecific KD of Gnmt, Sardh and CG5955 compare to age-matched control. N\#=9 from three flies' IFM per genotype. 


\section{Figure 3}
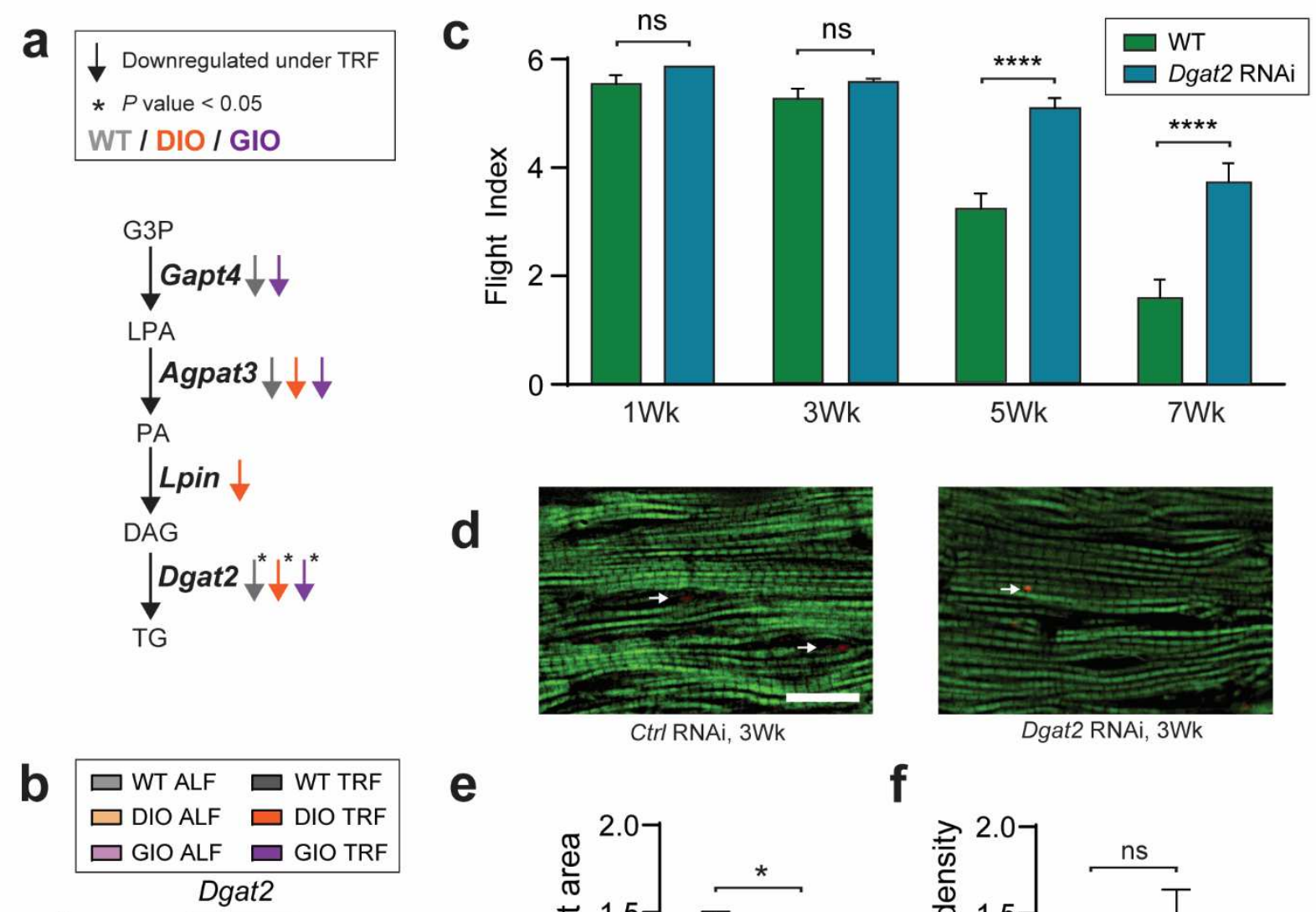

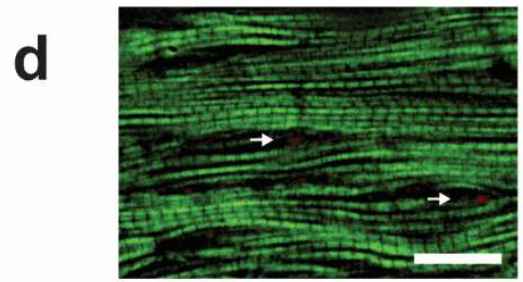

Ctrl RNAi, 3Wk

e

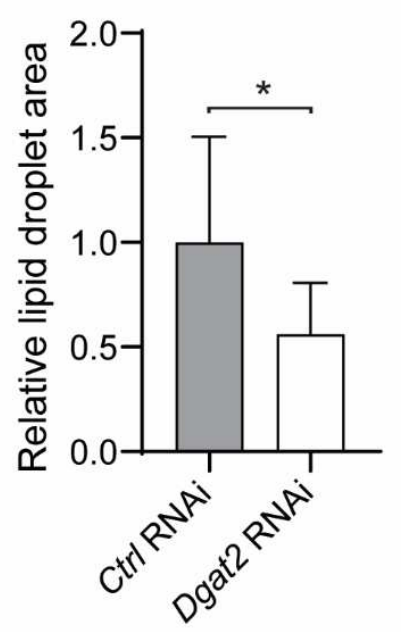

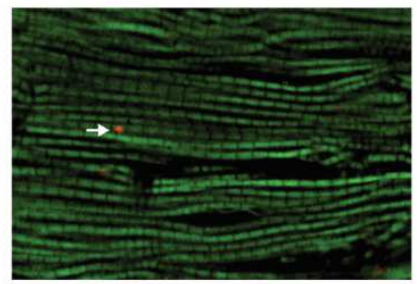

Dgat2 RNAi, 3Wk

f

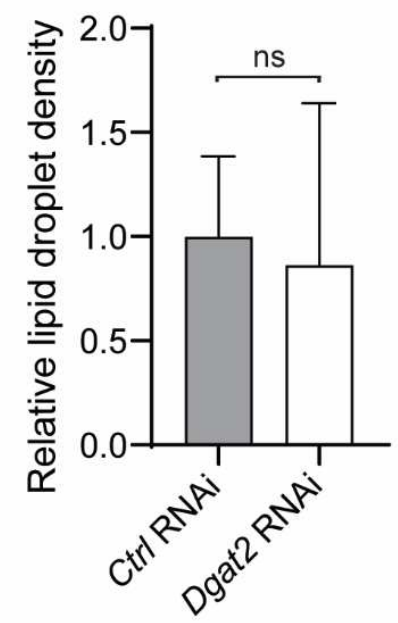

Figure 3: Gene expression and functional validation of Dgat2 in skeletal muscle in WT and obesity models.

(a) Schematic representation of de novo triacylglycerol synthesis with metabolite intermediates and important genes (italic bold) encoding enzymes. Down arrows indicate downregulation of gene expression and color indicates condition (grey WT, Orange DIO, purple GIO). Asterisk indicates p-value $<0.05$ and fold change $>1.25$.

(b) Expression level (normalized read count, log2) of gene Dgat2 under ALF and TRF in WT, DIO, GIO models.

(c) Flight performance of WT female flies with Dgat2 $K D$ at 1, 3, 5 and 7 weeks of age. N\# $=65-88$ female flies per time point. Bar graphs represent mean and SEM, with significance presented as ${ }^{\mathrm{ns}} p>0.05$, ${ }^{*} p \leq 0.05,{ }^{* *} p<0.01,{ }^{* * *} p<0.001$. 
(d) Fluorescence images of the IFM from 3-week-old females with IFM-specific $K D$ of Dgat2 upon probing with phalloidin (green) and Nile Red (red puncta). Smaller lipid (arrows) was detected in IFM of KD of Dgat2 (asterisks), compared with age-matched control. Scale bar is $20 \mu \mathrm{m}$.

(e-f) Intramuscular lipid quantification (size (e) and density (f)) showed significant reduction in lipid droplet area upon IFM-specific $K D$ of Dgat2 compare to age-matched control. $N \#=9$ from three flies' IFM per genotype. 
Figure 4

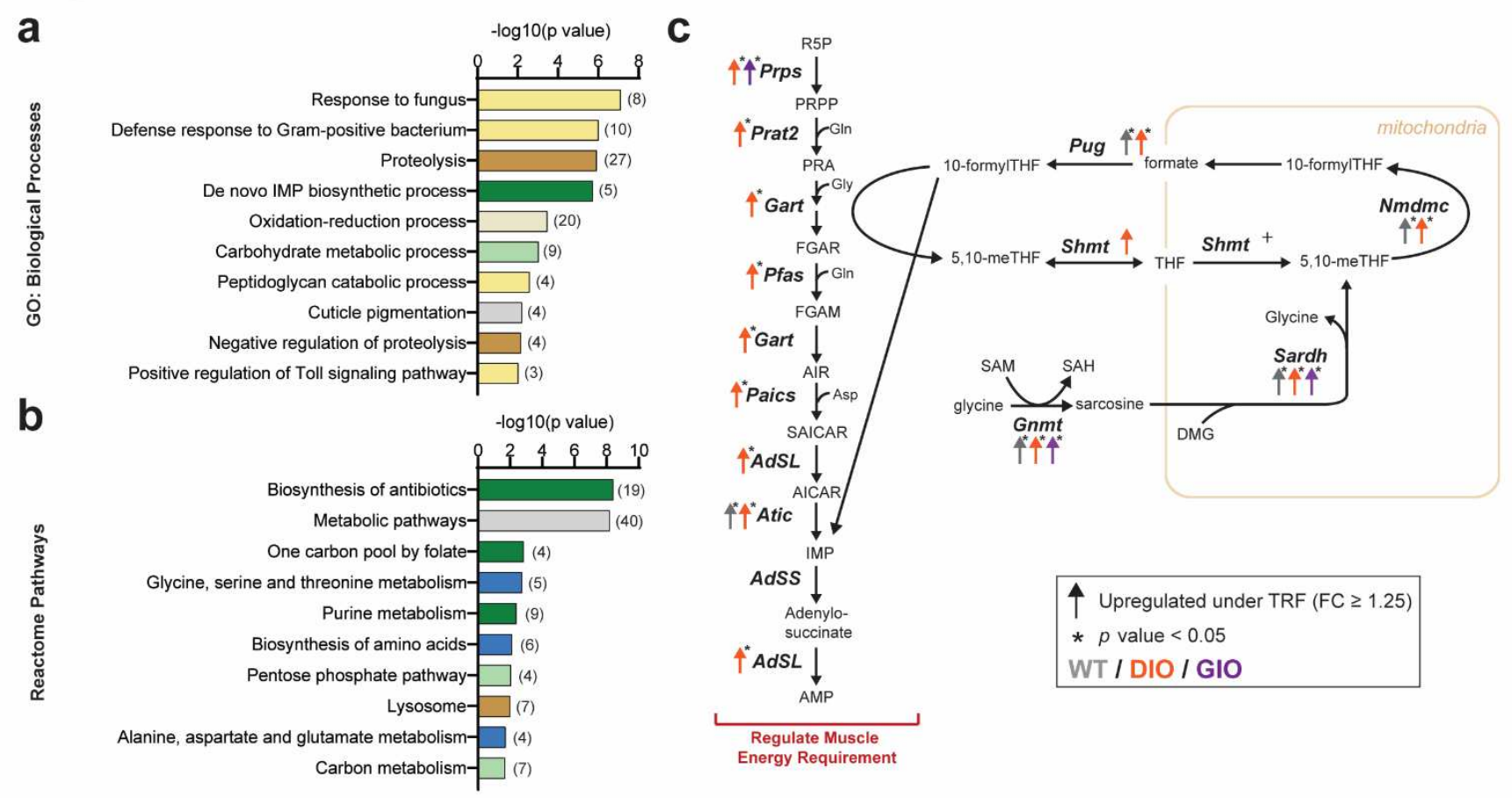

d

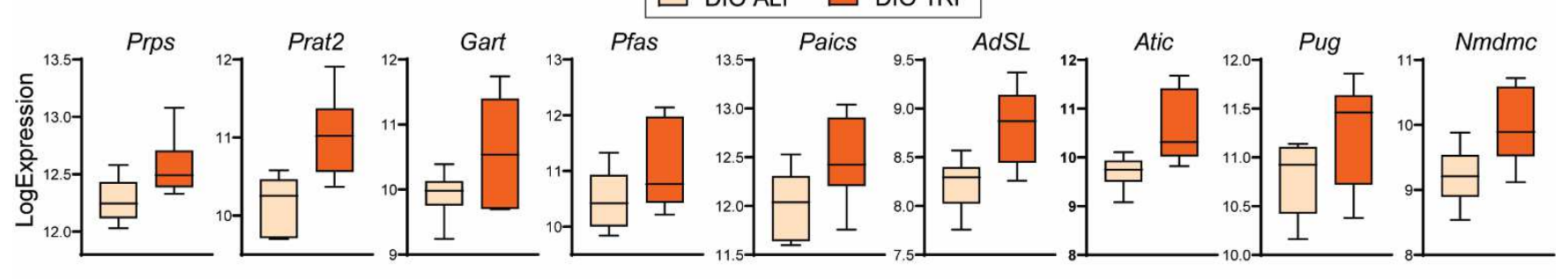

e
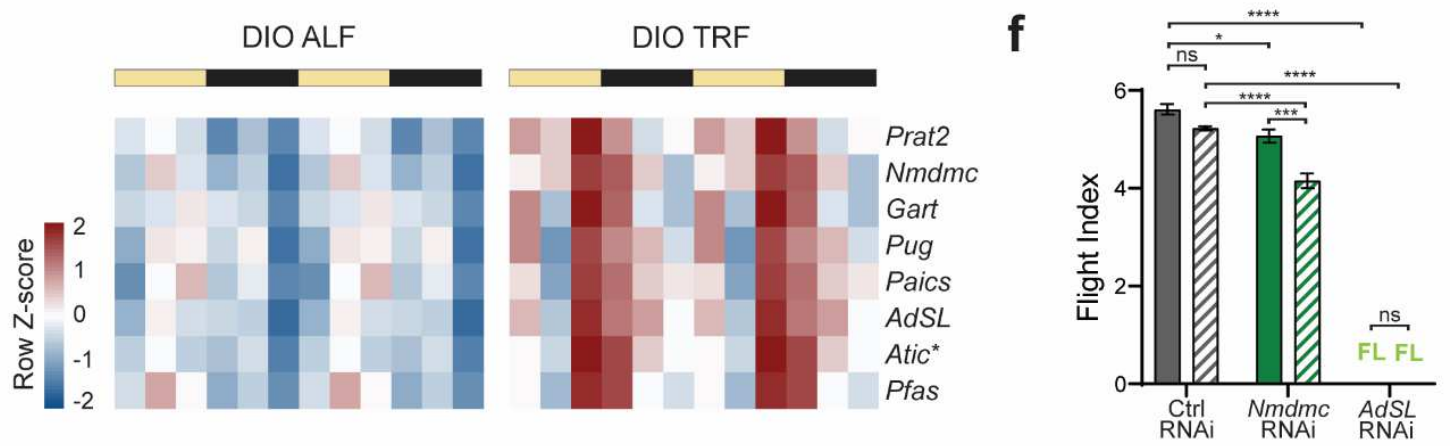

g
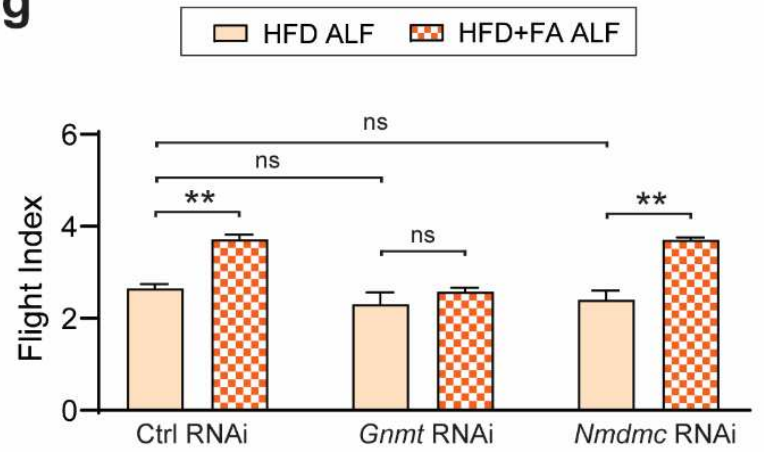

h

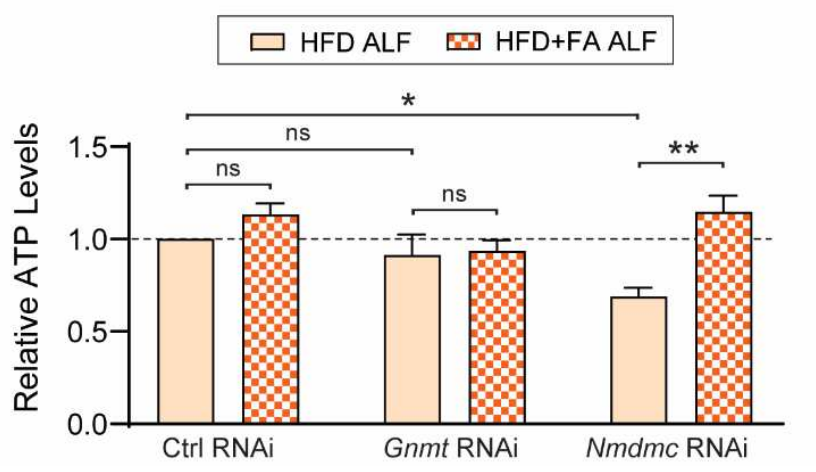


Figure 4: TRF upregulated genes linked with purine biosynthesis and folate cycle in DIO model. (a) GO analysis and (b) Reactome pathway analysis of 270 genes that were significantly upregulated under TRF in the DIO model. Bar charts represent the -log10 ( $p$ value) of each enriched GO term and pathway. Related pathways are colored similarly. The number of genes identified in each GO term and pathway is shown in parenthesis.

(c) Schematic representation of de novo purine biosynthesis and folate cycle with metabolic intermediates and important genes (italic bold) encoding enzymes possibly involved in energy regulation in muscle. Up arrows indicate upregulation of gene expression (fold change $\geq 1.25$ ), asterisk indicates $p$ value $\leq 0.05$ and color indicates condition (grey WT, Orange DIO, purple GIO).

(d) Expression level (normalized read count, log2) of significantly upregulated genes in de novo purine biosynthesis and folate cycle under TRF versus ALF in the DIO model.

(e) Heatmap representation of the genes in (d) with periodicity in the DIO model. Asterisk indicates the gain of periodicity under TRF.

(f) Flight performance of control, Nmdmc $K D$ and $A d S L K D$ at 1 and 3 weeks of age. FL indicates flightless. N\# = 50-70 per condition. FL indicates flightless. Error bars represent SEM. Bar graphs show mean and SEM, with significance presented as ${ }^{\mathrm{ns}} p>0.05,{ }^{*} p \leq 0.05,{ }^{* *} p<0.01,{ }^{* * *} p<0.001$.

(g) Flight performance of control, Gnmt KD and Nmdmc KD at 3 weeks of age under HFD ALF, and HFD+FA ALF. Error bars represent SEM. N\# 40-92 per condition. Bar graphs show mean and SEM, with significance presented as ${ }^{\mathrm{ns}} p>0.05,{ }^{*} p \leq 0.05,{ }^{* *} p<0.01,{ }^{* * *} p<0.001$.

(h) Relative ATP level measurement in control, IFM-specific KD of Gnmt and Nmdmc at 3 weeks of age under HFD ALF and HFD+FA ALF conditions with N\# = 3-5 per condition. Bar graphs represent mean and SEM, with significance presented as ${ }^{n s} p>0.05,{ }^{*} p \leq 0.05,{ }^{* *} p<0.01,{ }^{* * *} p<0.001$. 
Figure 5

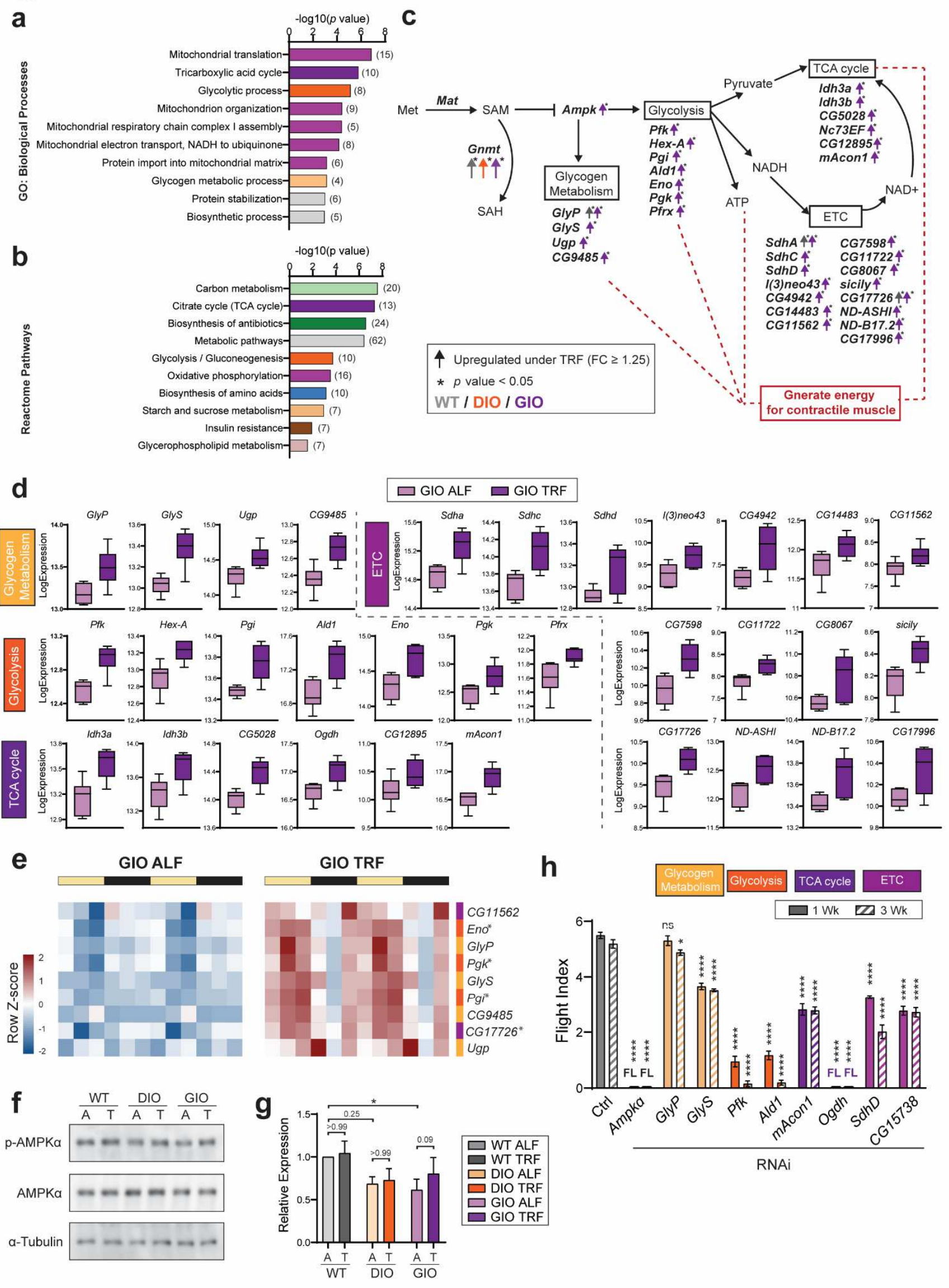


Figure 5: TRF upregulated genes associated with AMPK and downstream pathways in the GIO model.

(a) GO analysis and (b) Reactome pathway analysis of 408 genes that were significantly upregulated under TRF in the GIO model. Bar charts represent the -log10 ( $p$ value) of each enriched GO term and pathway. Related pathways are colored similarly. The number of genes identified in each GO term and pathway is shown in parenthesis.

(c) Schematic representation of connection between Gnmt, Ampk and downstream of AMPK signaling with important genes (italic bold) encoding enzymes. Up arrows indicate upregulation of gene expression (fold change $\geq 1.25$ ) and color indicates condition (grey WT, Orange DIO, purple GIO). Asterisk indicates $p$ value $\leq 0.05$ and fold change $\geq 1.25$. The up arrow of Ampk represents the expression of AMPK $\alpha$ subunit.

(d) Expression level (normalized read count, log2) of significantly upregulated genes in glycolysis, glycogen metabolism, TCA cycle and electron transport chain under TRF versus ALF in the GIO model.

(e) Heatmap representation of the genes in (d) with periodicity in the GIO model. Asterisk indicates the gain of periodicity under TRF.

(f) Representative western blot of p-AMPKa levels (top), AMPKa levels (middle) and $\alpha$-tubulin (bottom), from 3-week-old female fly IFMs in WT, DIO and GIO models under ALF (A) and TRF (T).

(g) Ratios of p-AMPKa/AMPKa normalized to WT ALF with N\# = 3 .

(h) Flight index of GIO genes in relation to downstream pathways shown at 1 and 3 weeks of age. N\#= 50-100 per condition. FL indicates flightless. Bar graphs represent mean and SEM, with significance presented as ${ }^{\mathrm{ns}} p>0.05,{ }^{*} p \leq 0.05,{ }^{* *} p<0.01,{ }^{* *} p<0.001$.

Figure 6

\section{Diet-Induced Obesity (DIO)}

\section{Genetic-Induced Obesity (GIO)}

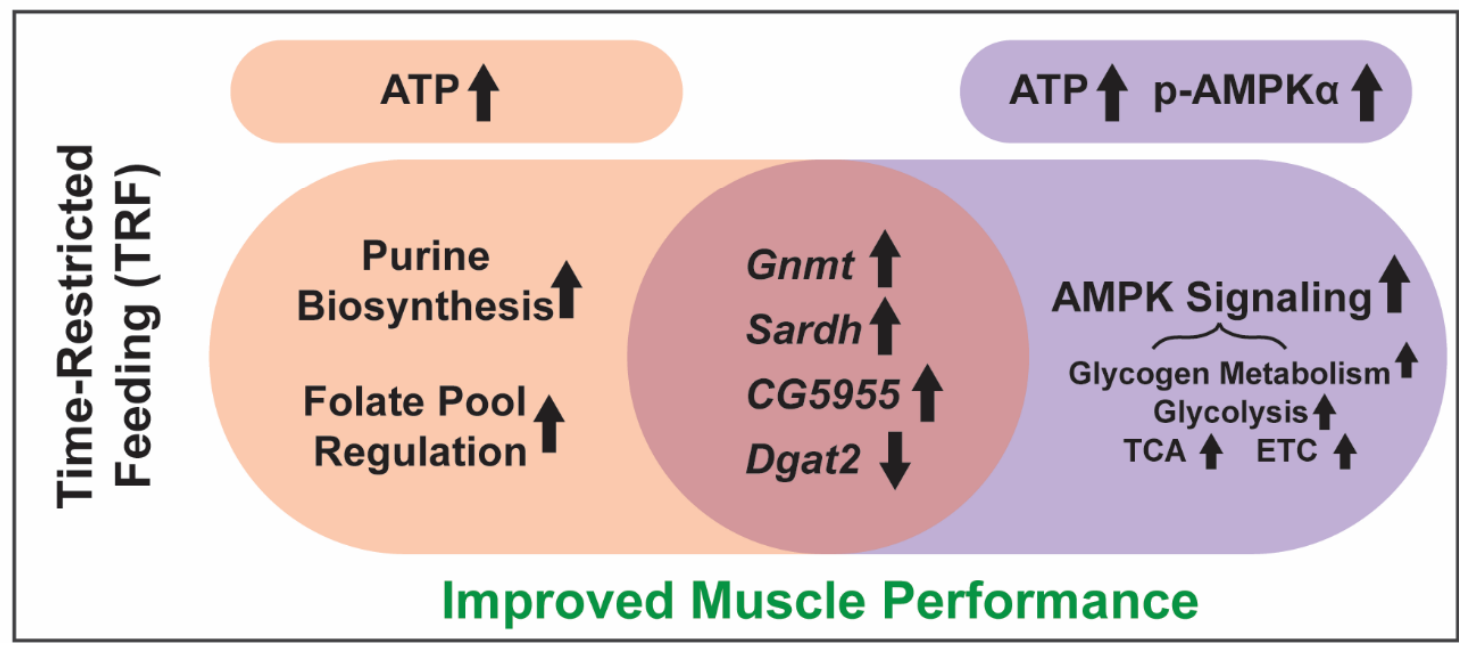

Figure 6: Proposed mechanism of TRF in Drosophila skeletal muscle under obesogenic challenges.

$12 \mathrm{~h}$ eating and $12 \mathrm{~h}$ fasting TRF intervention led to increased SAM regulation by upregulating of Gnmt, Sardh and CG5955, along with decreased TAG synthesis, which is supported by downregulation of Dgat2 in all conditions. In the diet-induced obesity model, TRF promoted purine biosynthesis and folate pool regulation, potentially resulting in higher ATP levels. However, in the genetic-induced obesity model, AMPK signaling, and downstream pathways were exclusively upregulated via increased $p$ AMPKa levels and increased ATP levels. All pathways regulated led to improved muscle performance in obesity models. 
Figure S1: Overview of transcriptome data in WT, DIO and GIO models

(a) PCA plot of skeletal muscle transcriptome data.

(b-d) Periodic skeletal muscle transcripts identified under ALF and TRF in WT (b), DIO (c) and GIO (d).

Figure S2: Periodicity of clock genes

(a-d) Expression level (normalized read count, log2) of Clk (a), Cyc (b), Tim (c) and Per (d) under ALF and TRF in WT, DIO and GIO.

(e-h) Temporal expressions of Clk (e), Cyc (f), Tim (g) and Per (h) under ALF and TRF in WT, DIO and GIO. Empirical_JTK periodicity detection was presented as a solid line (Corrected P-value $\leq 0.05$ ) or dash line (Corrected P-value > 0.05).

Figure S3: Profiles of the significantly upregulated genes across WT, DIO and GIO models

(a) A table of differentially expressed genes shared across WT, DIO and GIO models.

(b) Temporal expressions of Gnmt, Sardh and CG5955 under ALF and TRF in WT, DIO and GIO.

(c) Quantification of RNA levels from thoraces of 3-week-old flies following Gnmt, Sardh and CG5955 IFM-specific $K D$.

(d) Flight performance of WT male flies with Gnmt, Sardh or CG5955 IFM-specific KD at 1 week of age. Three independent RNAi lines per gene were tested. Combined average data were shown in green. N\# $=30-50$ females for each independent fly line per time point $(\mathrm{N} \#=100-150$ combined $)$. Bar graphs represent mean and SEM, with significance presented as ${ }^{\mathrm{ns}} p>0.05,{ }^{*} p \leq 0.05,{ }^{* *} p<0.01,{ }^{* * *} p<0.001$.

Figure S4: Profiles of the significantly downregulated genes across WT, DIO and GIO models (a) Temporal expressions of Dgat2 under ALF and TRF in WT, DIO and GIO.

(b) Expression level (normalized read count, log2) of Gpat4, Agpat3, and Lpin from de novo triacylglycerol synthesis under ALF and TRF in WT, DIO and GIO.

(c) Fluorescence images of the abdomen from 3-week-old females with IFM-specific KD of Dgat2 upon probing with Nile Red (red puncta). Scale bar is $20 \mu \mathrm{m}$.

(d-e) Lipid quantification (size (d) and density (e)) showed no significant differences in lipid droplet area upon IFM-specific $K D$ of $D g$ at2 compare to age-matched control. $N \#=9$ from three flies' abdomen per genotype.

(f) Expression profiles of Gla under ALF and TRF in WT, DIO and GIO.

(g) Flight performance of WT male flies with Gla IFM-specific $K D$ at 1, 3, 5 and 7 weeks of age. $N \#=67$ 99 females for each condition per time point. Bar graphs represent mean and SEM, with significance presented as ${ }^{\mathrm{ns}} p>0.05,{ }^{*} p \leq 0.05,{ }^{* *} p<0.01,{ }^{* *} p<0.001$.

Figure S5: GO and Reactome analyses of significantly downregulated genes under TRF versus ALF in obesity models.

(a) GO analysis and (b) Reactome pathway analysis of 636 genes that were significantly downregulated under TRF in the DIO model. Bar charts represent the - Log10 ( $p$ value) of each enriched GO term and pathway. The number of genes identified in each GO term and pathway is shown in parenthesis.

(c) GO analysis and (d) Reactome pathway analysis of 579 genes that were significantly downregulated under TRF in the GIO model. Bar charts represent the -Log10 ( $p$ value) of each enriched GO term and pathway. The number of genes identified in each GO term and pathway is shown in parenthesis.

Figure S6: De novo purine biosynthesis and folate cycle genes under ALF and TRF in WT, DIO and GIO models

(a) Expression levels of genes from Fig. 4d in WT, DIO and GIO 
(b) Heatmap representation of genes from Fig. 4e in WT and GIO models.

(c) Flight performance of 3-week-old female flies with IFM-specific KD of Gnmt and Nmdmc. N\# = 30-50 females for each condition. Bar graphs represent mean and SEM, with significance presented as ${ }^{\text {ns }} p>$ $0.05,{ }^{*} p \leq 0.05,{ }^{* *} p<0.01,{ }^{* * *} p<0.001$.

(d) Relative ATP levels of 3-week-old WT, DIO and GIO flies under ALF and TRF with N\# = 4.

(e) Flight performance of 10--day-old (left) and climbing performance of 10-day-old (right) DIO-ALF female flies with supplement of $0.1 \mathrm{mM}, 1 \mathrm{mM}, 4 \mathrm{mM}, 8 \mathrm{mM}$, and $16 \mathrm{mM}$ folic acid.

(f) Flight performance of 3-week-old WT, DIO and GIO female flies with or without folic acid supplement. $N \#=64-111$. Bar graphs represent mean and SEM, with significance presented as ${ }^{\mathrm{ns}} p>0.05,{ }^{*} p \leq 0.05$, ${ }^{* *} p<0.01,{ }^{* * *} p<0.001$.

(g) Climbing performance of 3-week-old WT, DIO and GIO female flies with or without folic acid supplement. $N \#=60-70$. Bar graphs represent mean and SEM, with significance presented as ${ }^{\mathrm{ns}} p>0.05$, ${ }^{*} p \leq 0.05,{ }^{* *} p<0.01,{ }^{* *} p<0.001$.

Figure S7: Glycolysis, glycogen metabolism, TCA cycle and ETC genes under ALF and TRF in WT, DIO and GIO models

(a) Expression of Ampka mRNA levels in WT, DIO and GIO models

(b) Expression levels of genes from Fig. 5d in WT, DIO and GIO

(c) Heatmap representation of the expression levels of purine biosynthesis associated genes in ALF and TRF in the WT and GIO model.

(d) Heatmap representation of the average expression levels of genes related to glycolysis, glycogen metabolism, TCA cycle, and ETC with increased but not significant expression under TRF versus ALF in the GIO model.

(e) Quantification of relative expression of western blot of AMPKa protein levels of WT, DIO and GIO under ALF and TRF, with $N \#=3$.

(f) Flight index of 3-week-old flies with indicated genotypes.

Figure S8: Comparison with skeletal muscle transcriptomic data from obese men under TRF. (a) Expression levels of genes associated with purine synthesis and folate cycle from Lundell et al., 2020 RNA-seq dataset under EXF and TRF.

(b) Expression levels of the same genes from (a) in our Drosophila RNA-seq dataset under ALF and TRF.

\section{Acknowledgement}

This work was supported by National Institutes of Health (NIH) grants AG065992 to GCM and AG068550 to GCM and SP. This work is also supported by UAB Startup funds 3123226 and 3123227 to GCM. We would like to thank Anju Melkani, UCSD for her help in dissection/isolation of muscle tissue and Jesús E. Villanueva, SDSU in implementing TRF. We would also like to thank Aniket Pant, UAB School of Medicine for his help with lipid quantification using Python. We also would like to thank Dr. Ralph Sanderson, Dr. Rakesh Patel and Mr. Jonathan Roth, UAB School of Medicine for their editorial comments on the manuscript. Stocks were obtained from the Bloomington Drosophila Stock Center and Vienna Drosophila Resource Center was used in this study.

Author contributions GCM and SP designed the experiments with the help from CL and YG. GCM, $\mathrm{CL}$, and HDL prepared sequencing samples. YG, HDL and $\mathrm{CL}$ analyzed transcriptomic data. CL, YG and SV performed experiments associated with WT, DIO, and GIO models. FA performed acquisition of cytological images and G.C.M analyzed lipid quantification. YG, CL and GCM prepared the paper with SP's input. All authors provided feedback on the manuscript. 


\section{References:}

1 Zlobine, I., Gopal, K. \& Ussher, J. R. Lipotoxicity in obesity and diabetes-related cardiac dysfunction. Biochim Biophys Acta 1861, 1555-1568, doi:10.1016/j.bbalip.2016.02.011 (2016).

$2 \quad$ Obesity: Its Causes and Treatment. Hospital (Lond 1886) 11, 46 (1891).

3 Shi, S. Q., Ansari, T. S., McGuinness, O. P., Wasserman, D. H. \& Johnson, C. H. Circadian disruption leads to insulin resistance and obesity. Curr Biol 23, 372-381, doi:10.1016/j.cub.2013.01.048 (2013).

4 Tallis, J., James, R. S. \& Seebacher, F. The effects of obesity on skeletal muscle contractile function. J Exp Biol 221, doi:10.1242/jeb.163840 (2018).

$5 \quad \mathrm{Li}, \mathrm{Y}$., Xu, S., Zhang, X., Yi, Z. \& Cichello, S. Skeletal intramyocellular lipid metabolism and insulin resistance. Biophys Rep 1, 90-98, doi:10.1007/s41048-015-0013-0 (2015).

6 Jensen, J., Rustad, P. I., Kolnes, A. J. \& Lai, Y. C. The role of skeletal muscle glycogen breakdown for regulation of insulin sensitivity by exercise. Front Physiol 2, 112, doi:10.3389/fphys.2011.00112 (2011).

7 Goodpaster, B. H., He, J., Watkins, S. \& Kelley, D. E. Skeletal muscle lipid content and insulin resistance: evidence for a paradox in endurance-trained athletes. J Clin Endocrinol Metab 86, 5755-5761, doi:10.1210/jcem.86.12.8075 (2001).

8 Elshorbagy, A. K., Jerneren, F., Samocha-Bonet, D., Refsum, H. \& Heilbronn, L. K. Serum Sadenosylmethionine, but not methionine, increases in response to overfeeding in humans. Nutr Diabetes 6, e192, doi:10.1038/nutd.2015.44 (2016).

9 Ducker, G. S. \& Rabinowitz, J. D. One-Carbon Metabolism in Health and Disease. Cell Metab 25, 27-42, doi:10.1016/j.cmet.2016.08.009 (2017).

10 Chaix, A., Lin, T., Le, H. D., Chang, M. W. \& Panda, S. Time-Restricted Feeding Prevents Obesity and Metabolic Syndrome in Mice Lacking a Circadian Clock. Cell Metab 29, 303-319 e304, doi:10.1016/j.cmet.2018.08.004 (2019).

11 Villanueva, J. E. et al. Time-restricted feeding restores muscle function in Drosophila models of obesity and circadian-rhythm disruption. Nat Commun 10, 2700, doi:10.1038/s41467-01910563-9 (2019).

12 Barber, A. F., Erion, R., Holmes, T. C. \& Sehgal, A. Circadian and feeding cues integrate to drive rhythms of physiology in Drosophila insulin-producing cells. Genes Dev 30, 2596-2606, doi:10.1101/gad.288258.116 (2016).

13 Hatori, M. et al. Time-restricted feeding without reducing caloric intake prevents metabolic diseases in mice fed a high-fat diet. Cell Metab 15, 848-860, doi:10.1016/j.cmet.2012.04.019 (2012).

14 Lundell, L. S. et al. Time-restricted feeding alters lipid and amino acid metabolite rhythmicity without perturbing clock gene expression. Nat Commun 11, 4643, doi:10.1038/s41467-02018412-w (2020).

15 Parr, E. B., Devlin, B. L., Radford, B. E. \& Hawley, J. A. A Delayed Morning and Earlier Evening Time-Restricted Feeding Protocol for Improving Glycemic Control and Dietary Adherence in Men with Overweight/Obesity: A Randomized Controlled Trial. Nutrients 12, doi:10.3390/nu12020505 (2020).

16 McAllister, M. J., Pigg, B. L., Renteria, L. I. \& Waldman, H. S. Time-restricted feeding improves markers of cardiometabolic health in physically active college-age men: a 4-week randomized pre-post pilot study. Nutr Res 75, 32-43, doi:10.1016/j.nutres.2019.12.001 (2020).

17 Gill, S., Le, H. D., Melkani, G. C. \& Panda, S. Time-restricted feeding attenuates age-related cardiac decline in Drosophila. Science 347, 1265-1269, doi:10.1126/science.1256682 (2015). Chatterjee, N. \& Perrimon, N. What fuels the fly: Energy metabolism in Drosophila and its application to the study of obesity and diabetes. Sci Adv 7, doi:10.1126/sciadv.abg4336 (2021). 
Demontis, F., Piccirillo, R., Goldberg, A. L. \& Perrimon, N. Mechanisms of skeletal muscle aging: insights from Drosophila and mammalian models. Dis Model Mech 6, 1339-1352, doi:10.1242/dmm.012559 (2013).

20 Wang, Y. et al. Expression of the inclusion body myopathy 3 mutation in Drosophila depresses myosin function and stability and recapitulates muscle inclusions and weakness. Mol Biol Cell 23, 2057-2065, doi:10.1091/mbc.E12-02-0120 (2012).

21 Birse, R. T. et al. High-fat-diet-induced obesity and heart dysfunction are regulated by the TOR pathway in Drosophila. Cell Metab 12, 533-544, doi:10.1016/j.cmet.2010.09.014 (2010).

22 Walls, S. M., Jr. et al. Identification of sphingolipid metabolites that induce obesity via misregulation of appetite, caloric intake and fat storage in Drosophila. PLoS Genet 9 , e1003970, doi:10.1371/journal.pgen.1003970 (2013).

23 Walls, S. M. et al. Ceramide-Protein Interactions Modulate Ceramide-Associated Lipotoxic Cardiomyopathy. Cell Rep 22, 2702-2715, doi:10.1016/j.celrep.2018.02.034 (2018).

24 Arinze, I. J. Facilitating understanding of the purine nucleotide cycle and the one-carbon pool: Part I: The purine nucleotide cycle. Biochem Mol Biol Educ 33, 165-168, doi:10.1002/bmb.2005.494033032469 (2005).

25 Flanagan, W. F., Holmes, E. W., Sabina, R. L. \& Swain, J. L. Importance of purine nucleotide cycle to energy production in skeletal muscle. Am J Physiol 251, C795-802, doi:10.1152/ajpcell.1986.251.5.C795 (1986).

26 Chang, E. \& Kim, Y. Vitamin D Ameliorates Fat Accumulation with AMPK/SIRT1 Activity in C2C12 Skeletal Muscle Cells. Nutrients 11, doi:10.3390/nu11112806 (2019).

27 Guevara-Cruz, M. et al. Genistein stimulates insulin sensitivity through gut microbiota reshaping and skeletal muscle AMPK activation in obese subjects. BMJ Open Diabetes Res Care 8, doi:10.1136/bmjdrc-2019-000948 (2020).

28 Love, M. I., Huber, W. \& Anders, S. Moderated estimation of fold change and dispersion for RNA-seq data with DESeq2. Genome Biol 15, 550, doi:10.1186/s13059-014-0550-8 (2014). Hutchison, A. L. et al. Improved statistical methods enable greater sensitivity in rhythm detection for genome-wide data. PLoS Comput Biol 11, e1004094, doi:10.1371/journal.pcbi.1004094 (2015).

30 Shyh-Chang, N. et al. Influence of threonine metabolism on S-adenosylmethionine and histone methylation. Science 339, 222-226, doi:10.1126/science.1226603 (2013).

31 Jacquier, N. et al. Lipid droplets are functionally connected to the endoplasmic reticulum in Saccharomyces cerevisiae. J Cell Sci 124, 2424-2437, doi:10.1242/jcs.076836 (2011).

32 Kuerschner, L., Moessinger, C. \& Thiele, C. Imaging of lipid biosynthesis: how a neutral lipid enters lipid droplets. Traffic 9, 338-352, doi:10.1111/j.1600-0854.2007.00689.x (2008).

33 Yen, C. L., Stone, S. J., Koliwad, S., Harris, C. \& Farese, R. V., Jr. Thematic review series: glycerolipids. DGAT enzymes and triacylglycerol biosynthesis. J Lipid Res 49, 2283-2301, doi:10.1194/jlr.R800018-JLR200 (2008).

$34 \quad$ Wilfling, F. et al. Triacylglycerol synthesis enzymes mediate lipid droplet growth by relocalizing from the ER to lipid droplets. Dev Cell 24, 384-399, doi:10.1016/j.devcel.2013.01.013 (2013).

35 Wang, W. et al. Glycine metabolism in animals and humans: implications for nutrition and health. Amino Acids 45, 463-477, doi:10.1007/s00726-013-1493-1 (2013).

36 French, J. B. et al. Spatial colocalization and functional link of purinosomes with mitochondria. Science 351, 733-737, doi:10.1126/science.aac6054 (2016).

37 Fustin, J. M. et al. Rhythmic nucleotide synthesis in the liver: temporal segregation of metabolites. Cell Rep 1, 341-349, doi:10.1016/j.celrep.2012.03.001 (2012).

38 Thurley, K. et al. Principles for circadian orchestration of metabolic pathways. Proc Natl Acad Sci U S A 114, 1572-1577, doi:10.1073/pnas.1613103114 (2017).

39 Ishikawa, K. \& Shimazu, T. Daily rhythms of glycogen synthetase and phosphorylase activities in rat liver: influence of food and light. Life Sci 19, 1873-1878, doi:10.1016/0024-

3205(76)90119-3 (1976). 
Ishikawa, K. \& Shimazu, T. Circadian rhythm of liver glycogen metabolism in rats: effects of hypothalamic lesions. Am J Physiol 238, E21-25, doi:10.1152/ajpendo.1980.238.1.E21 (1980).

41 Akimoto, H., Kinumi, T. \& Ohmiya, Y. Circadian rhythm of a TCA cycle enzyme is apparently regulated at the translational level in the dinoflagellate Lingulodinium polyedrum. $\mathrm{J}$ Biol Rhythms 20, 479-489, doi:10.1177/0748730405280811 (2005). Neufeld-Cohen, A. et al. Circadian control of oscillations in mitochondrial rate-limiting enzymes and nutrient utilization by PERIOD proteins. Proc Natl Acad Sci U S A 113, E1673-1682, doi:10.1073/pnas.1519650113 (2016).

43 Melkani, G. C. \& Panda, S. Time-restricted feeding for prevention and treatment of cardiometabolic disorders. J Physiol 595, 3691-3700, doi:10.1113/JP273094 (2017).

44 Obata, F. \& Miura, M. Enhancing S-adenosyl-methionine catabolism extends Drosophila lifespan. Nat Commun 6, 8332, doi:10.1038/ncomms9332 (2015).

45 Harris, C. A. et al. DGAT enzymes are required for triacylglycerol synthesis and lipid droplets in adipocytes. J Lipid Res 52, 657-667, doi:10.1194/jlr.M013003 (2011).

46 Frezza, M., Centini, G., Cammareri, G., Le Grazie, C. \& Di Padova, C. S-adenosylmethionine for the treatment of intrahepatic cholestasis of pregnancy. Results of a controlled clinical trial. Hepatogastroenterology 37 Suppl 2, 122-125 (1990).

47 Lu, S. C. \& Mato, J. M. S-adenosylmethionine in liver health, injury, and cancer. Physiol Rev 92, 1515-1542, doi:10.1152/physrev.00047.2011 (2012).

48 Qin, B. et al. A trial of ademetionine in the treatment of intrahepatic biliary stasis viral hepatitis. Zhonghua Gan Zang Bing Za Zhi 8, 158-160 (2000).

$49 \mathrm{Gu}, \mathrm{X}$. et al. SAMTOR is an S-adenosylmethionine sensor for the mTORC1 pathway. Science 358, 813-818, doi:10.1126/science.aao3265 (2017).

50 Khamzina, L., Veilleux, A., Bergeron, S. \& Marette, A. Increased activation of the mammalian target of rapamycin pathway in liver and skeletal muscle of obese rats: possible involvement in obesity-linked insulin resistance. Endocrinology 146, 1473-1481, doi:10.1210/en.2004-0921 (2005).

51 Tremblay, F. \& Marette, A. Amino acid and insulin signaling via the mTOR/p70 S6 kinase pathway. A negative feedback mechanism leading to insulin resistance in skeletal muscle cells. J Biol Chem 276, 38052-38060, doi:10.1074/jbc.M106703200 (2001). Elshorbagy, A. K. et al. S-adenosylmethionine is associated with fat mass and truncal adiposity in older adults. J Nutr 143, 1982-1988, doi:10.3945/jn.113.179192 (2013).

53 Huang, J. W., Chen, C. J., Yen, C. H., Chen, Y. A. \& Liu, Y. P. Loss of Glycine NMethyltransferase Associates with Angiopoietin-Like Protein 8 Expression in High Fat-Diet-Fed Mice. Int J Mol Sci 20, doi:10.3390/ijms20174223 (2019).

54 Liao, Y. J. et al. Glycine N-methyltransferase deficiency in female mice impairs insulin signaling and promotes gluconeogenesis by modulating the PI3K/Akt pathway in the liver. J Biomed Sci 23, 69, doi:10.1186/s12929-016-0278-8 (2016).

55 Edgar, A. J. The human L-threonine 3-dehydrogenase gene is an expressed pseudogene. BMC Genet 3, 18, doi:10.1186/1471-2156-3-18 (2002).

56 Levin, M. C. et al. Increased lipid accumulation and insulin resistance in transgenic mice expressing DGAT2 in glycolytic (type II) muscle. Am J Physiol Endocrinol Metab 293, E17721781, doi:10.1152/ajpendo.00158.2007 (2007).

57 Tullson, P. C. \& Terjung, R. L. Adenine nucleotide metabolism in contracting skeletal muscle. Exerc Sport Sci Rev 19, 507-537 (1991).

58 Salerno, C., lotti, S., Lodi, R., Crifo, C. \& Barbiroli, B. Failure of muscle energy metabolism in a patient with adenylosuccinate lyase deficiency. An in vivo study by phosphorus NMR spectroscopy. Biochim Biophys Acta 1360, 271-276, doi:10.1016/s0925-4439(97)00010-0 (1997).

$59 \mathrm{Li}$, J. et al. Association between Serum Folate and Insulin Resistance among U.S. Nondiabetic Adults. Sci Rep 7, 9187, doi:10.1038/s41598-017-09522-5 (2017). 
60 Blom, H. J. \& Smulders, Y. Overview of homocysteine and folate metabolism. With special references to cardiovascular disease and neural tube defects. J Inherit Metab Dis 34, 75-81, doi:10.1007/s10545-010-9177-4 (2011).

61 Czeizel, A. E., Dudas, I., Vereczkey, A. \& Banhidy, F. Folate deficiency and folic acid supplementation: the prevention of neural-tube defects and congenital heart defects. Nutrients 5, 4760-4775, doi:10.3390/nu5114760 (2013).

62 Pena, J. \& Claro, J. C. [Is folic acid effective for the prevention of cardiovascular events in patients with advanced or terminal chronic kidney disease?]. Rev Med Chil 142, 636-645, doi:10.4067/S0034-98872014000500013 (2014).

63 Wang, Y. C. et al. A novel role of the tumor suppressor GNMT in cellular defense against DNA damage. Int J Cancer 134, 799-810, doi:10.1002/ijc.28420 (2014).

64 Simile, M. M. et al. Alterations of methionine metabolism in hepatocarcinogenesis: the emergent role of glycine N-methyltransferase in liver injury. Ann Gastroenterol 31, 552-560, doi:10.20524/aog.2018.0288 (2018).

65 Herzig, S. \& Shaw, R. J. AMPK: guardian of metabolism and mitochondrial homeostasis. Nat Rev Mol Cell Biol 19, 121-135, doi:10.1038/nrm.2017.95 (2018).

66 Hunter, R. W., Treebak, J. T., Wojtaszewski, J. F. \& Sakamoto, K. Molecular mechanism by which AMP-activated protein kinase activation promotes glycogen accumulation in muscle. Diabetes 60, 766-774, doi:10.2337/db10-1148 (2011).

67 Sinnett, S. E. \& Brenman, J. E. The Role of AMPK in Drosophila melanogaster. Exp Supp/ 107, 389-401, doi:10.1007/978-3-319-43589-3_16 (2016).

68 Janzen, N. R., Whitfield, J. \& Hoffman, N. J. Interactive Roles for AMPK and Glycogen from Cellular Energy Sensing to Exercise Metabolism. Int J Mol Sci 19, doi:10.3390/ijms19113344 (2018).

69 Giordano, F. J. Oxygen, oxidative stress, hypoxia, and heart failure. J Clin Invest 115, 500-508, doi:10.1172/JCl24408 (2005).

70 Gnaiger, E. Capacity of oxidative phosphorylation in human skeletal muscle: new perspectives of mitochondrial physiology. Int J Biochem Cell Biol 41, 1837-1845, doi:10.1016/j.biocel.2009.03.013 (2009).

71 Joseph, B. K. et al. Inhibition of AMP Kinase by the Protein Phosphatase 2A Heterotrimer, PP2APpp2r2d. J Biol Chem 290, 10588-10598, doi:10.1074/jbc.M114.626259 (2015).

72 Paolini, A. et al. Attenuation of autophagy impacts on muscle fibre development, starvation induced stress and fibre regeneration following acute injury. Sci Rep 8, 9062, doi:10.1038/s41598-018-27429-7 (2018).

73 Ljubicic, V. et al. Chronic AMPK activation evokes the slow, oxidative myogenic program and triggers beneficial adaptations in mdx mouse skeletal muscle. Hum Mol Genet 20, 3478-3493, doi:10.1093/hmg/ddr265 (2011).

74 Bergeron, R. et al. Chronic activation of AMP kinase results in NRF-1 activation and mitochondrial biogenesis. Am J Physiol Endocrinol Metab 281, E1340-1346, doi:10.1152/ajpendo.2001.281.6.E1340 (2001).

75 Drummond, D. R., Hennessey, E. S. \& Sparrow, J. C. Characterisation of missense mutations in the Act88F gene of Drosophila melanogaster. Mol Gen Genet 226, 70-80, doi:10.1007/BF00273589 (1991). 


\section{Glossary of metabolite abbrevations and Drosophila orthologs of human genes}

\begin{tabular}{|l|l|}
\hline Abbreviation & Metabolite \\
\hline SAM & S-adenosylmethionine \\
\hline sarcosine & N-methylglycine \\
\hline SAH & S-adenosylhomocysteine \\
\hline G3P & Glycerol 3-phosphate \\
\hline LPA & Lysophosphatidic acid \\
\hline PA & Phosphatidic acid \\
\hline DAG & Diacrylglycerol \\
\hline TG & Triacylglycerol \\
\hline DMG & Dimethylglycine \\
\hline IMP & Inosine 5'-monophosphate \\
\hline Met & Methionine \\
\hline R5P & Ribose 5-phosphate \\
\hline PRPP & 5-phospho-alpha-D-ribose 1-diphosphate \\
\hline PRA & 5-phosphoribosylamine \\
\hline FGAR & 5'-phosphoribosylformylglycinamide \\
\hline FGAM & 5'-phosphoribosylformylglycinamidine \\
\hline
\end{tabular}




\begin{tabular}{|l|l|}
\hline AIR & 5'-phosphoribodyl-5-aminoimidazole \\
\hline SAICAR & $\begin{array}{l}\text { 5'-phosphoribosyl-5-aminomidazole-4-N- } \\
\text { succinocarboxamide }\end{array}$ \\
\hline AICAR & 5'-phosphoribosyl-5-aminoimidazole-4-carboxamide \\
\hline IMP & Inosine 5'-monophosphate \\
\hline AMP & Adenosine monophosphate \\
\hline XMP & Xanthylate \\
\hline GMP & Guanylate \\
\hline THF & Tetrahydrofolate \\
\hline
\end{tabular}

\begin{tabular}{|c|c|c|}
\hline $\begin{array}{l}\text { Drosophila } \\
\text { Ortholog }\end{array}$ & $\begin{array}{l}\text { Human } \\
\text { Gene }\end{array}$ & Function Annotation \\
\hline CG6188/Gnmt & Gnmt & Glycine N-methyltransferase \\
\hline CG6385/Sardh & Sardh & Sarcosine dehydrogenase \\
\hline CG5995 & Tdh & threonine dehydrogenase \\
\hline CG6806/Lsp2 & N/A & Larval serum protein 2 \\
\hline CG5896/grass & N/A & Gram-positive specific serine protease \\
\hline CG1942/Dgat2 & Dgat2 & Diacylglycerol O-acyltransferase 2 \\
\hline CG7997 & $\mathrm{N} / \mathrm{A}$ & Predicted to have alpha-galactosidase activity \\
\hline CG13992 & N/A & Unknown \\
\hline CG10361 & GCAT & glycine C-acetyltransferase \\
\hline CG3209/Gpat4 & Gpat4 & Glycerol-3-phosphate O-acyltransferase 4 \\
\hline CG4729/Agpat3 & Agpat 3 & 1-Acylglycerol-3-phosphate O-acyltransferase 3 \\
\hline CG8709/Lpin & Lpin3 & Lipin \\
\hline CG3011/Shmt & Shmt1/2 & Serine hydroxymethyl transferase \\
\hline CG4067/pug & Mthfd1 & $\begin{array}{l}\text { Trifunctional enzyme methylenetetrahydrofolate } \\
\text { dehydrogenase involved in the pigmentation of pteridines } \\
\text { and ommochromes }\end{array}$ \\
\hline CG18466/Nmdmc & Mthfd2 & NAD-dependent methylenetetrahydrofolate dehydrogenase \\
\hline CG6767/Prps & Prps1 & Phosphoribosyl pyrophosphate synthetase \\
\hline CG10078/Prat2 & Ppat & Phosphoribosylamidotransferase 2 \\
\hline CG31628/Gart & Gart & GART trifunctional enzyme \\
\hline CG9127/Pfas & Pfas & Phosphoribosylformylglycinamidine synthase \\
\hline CG3989/Paics & Paics & PAICS bifunctional enzyme \\
\hline CG3590/AdSL & Adsl & Adenylosuccinate lyase \\
\hline CG11089 & Atic & $\begin{array}{l}\text { Predicted to have IMP cyclohydrolase activity and } \\
\text { phosphoribosylaminoimidazolecarboxamide } \\
\text { formyltransferase activity }\end{array}$ \\
\hline CG17273/AdSS & Adss & Adenylosuccinate synthase \\
\hline CG1799/ras & Impdh1 & Predicted to have IMP dehydrogenase activity. \\
\hline CG9242/bur & Gmps & Exhibits deubiquitinase activator activity. \\
\hline CG3051/AMPKa & Ampka & AMP-activated protein kinase $\alpha$ subunit \\
\hline CG7254/GlyP & Pygm & Glycogen phosphorylase \\
\hline CG6904/GlyS & Gys1 & Glycogen synthase \\
\hline
\end{tabular}




\begin{tabular}{|c|c|c|}
\hline CG4347/Ugp & Ugp2 & UDP-glucose pyrophosphorylase \\
\hline CG9485 & Agl & $\begin{array}{l}\text { Predicted to have } 4 \text {-alpha-glucanotransferase activity and } \\
\text { amylo-alpha-1,6-glucosidase activity }\end{array}$ \\
\hline CG5165/Pgm1 & Pgm & Phosphoglucose mutase 1 \\
\hline CG33138/AGBE & Gbe1 & 1,4-Alpha-Glucan Branching Enzyme \\
\hline CG4001/Pfk & Pfkm & phosphofructokinase \\
\hline CG3001/Hex-A & $\mathrm{Hk} 2$ & Hexokinase A \\
\hline CG8251/Pgi & Gpi & Phosphoglucose isomerase \\
\hline CG6058/Ald1 & Aldoc & Aldolase 1 \\
\hline CG17654/Eno & Eno1 & Enolase \\
\hline CG3127/Pgk & Pgk1 & Phosphoglycerate kinase \\
\hline CG3400/pfrx & Pfkfb1 & 6-phosphofructo-2-kinase \\
\hline CG12233/Idh3a & $\operatorname{Idh} 3 a$ & Isocitrate dehydrogenase $3 a$ \\
\hline CG6439/Idh3b & Idh3b & Isocitrate dehydrogenase $3 \mathrm{~b}$ \\
\hline CG5028 & Idh3g & Predicted to have isocitrate dehydrogenase (NAD+) activity \\
\hline CG11661/Ogdh & Ogdh & $\begin{array}{l}\text { Predicted to have oxoglutarate dehydrogenase (succinyl- } \\
\text { transferring) activity }\end{array}$ \\
\hline CG12895 & Sdhaf2 & $\begin{array}{l}\text { Predicted to be involved in cellular respiration; } \\
\text { mitochondrial respiratory chain complex II assembly; and } \\
\text { protein-FAD linkage. }\end{array}$ \\
\hline CG9244/mAcon1 & Aco2 & Mitochondrial aconitase 1 \\
\hline CG17246/SdhA & Sdha & Succinate dehydrogenase, subunit $A$ \\
\hline CG3283/SdhB & Sdhb & Succinate dehydrogenase, subunit $B$ \\
\hline CG6666/SdhC & Sdhc & Succinate dehydrogenase, subunit $\mathrm{C}$ \\
\hline CG10219/SdhD & Sdhd & Succinate dehydrogenase, subunit $D$ \\
\hline CG14865/I(3)neo43 & Cox16 & $\begin{array}{l}\text { Predicted to be involved in mitochondrial cytochrome c } \\
\text { oxidase assembly }\end{array}$ \\
\hline CG4942 & Cox18 & $\begin{array}{l}\text { Predicted to be involved in mitochondrial cytochrome c } \\
\text { oxidase assembly }\end{array}$ \\
\hline CG14483 & Pet100 & $\begin{array}{l}\text { Predicted to be involved in mitochondrial cytochrome c } \\
\text { oxidase assembly }\end{array}$ \\
\hline CG11562 & Pet117 & $\begin{array}{l}\text { Predicted to be involved in mitochondrial cytochrome } \mathrm{c} \\
\text { oxidase assembly }\end{array}$ \\
\hline CG7598/CIA30 & Ndufaf1 & $\begin{array}{l}\text { Predicted to have unfolded protein binding activity. Involved } \\
\text { in several processes, including mitochondrial electron } \\
\text { transport, NADH to ubiquinone; mitochondrial respiratory } \\
\text { chain complex I assembly }\end{array}$ \\
\hline CG11722 & Ndufaf4 & $\begin{array}{l}\text { Predicted to be involved in mitochondrial respiratory chain } \\
\text { complex I assembly }\end{array}$ \\
\hline CG8067 & Ndufaf5 & $\begin{array}{l}\text { Predicted to be involved in mitochondrial respiratory chain } \\
\text { complex I assembly }\end{array}$ \\
\hline CG15738/sicily & Ndufaf6 & Severe impairment of $\mathrm{Cl}$ with lengthened youth \\
\hline CG17726 & Ndufaf7 & $\begin{array}{l}\text { Predicted to have protein-arginine omega-N symmetric } \\
\text { methyltransferase activity. Predicted to be involved in } \\
\text { mitochondrial respiratory chain complex I assembly. }\end{array}$ \\
\hline CG3192/ND-ASHI & Ndufb8 & NADH dehydrogenase (ubiquinone) ASHI subunit \\
\hline
\end{tabular}




\begin{tabular}{|l|l|l|} 
CG3214/ND-B17.2 & Ndufa12 & Predicted to have NADH dehydrogenase activity \\
\hline CG17996 & Cmc1 & $\begin{array}{l}\text { Predicted to have copper ion binding activity. Predicted to } \\
\text { be involved in mitochondrial cytochrome c oxidase } \\
\text { assembly }\end{array}$ \\
\hline
\end{tabular}


Figure 1

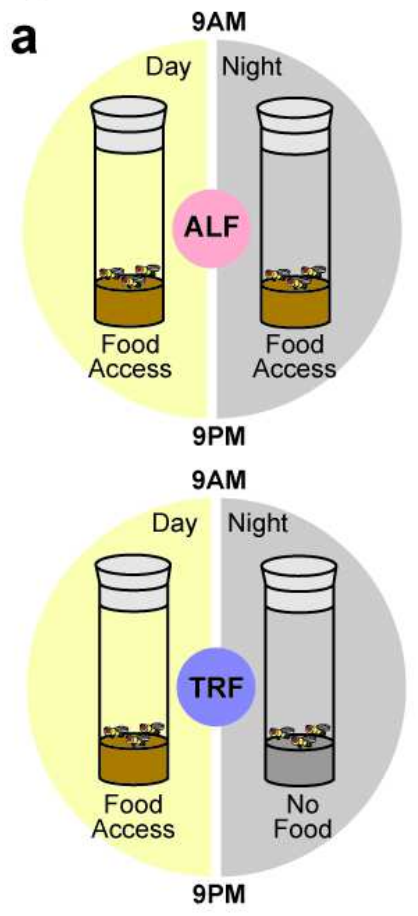

C Up Genes under TRF

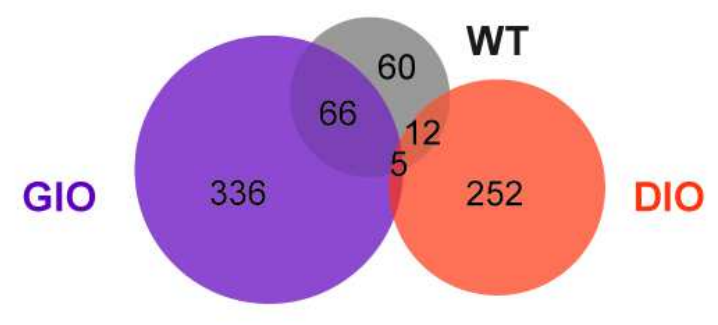

e

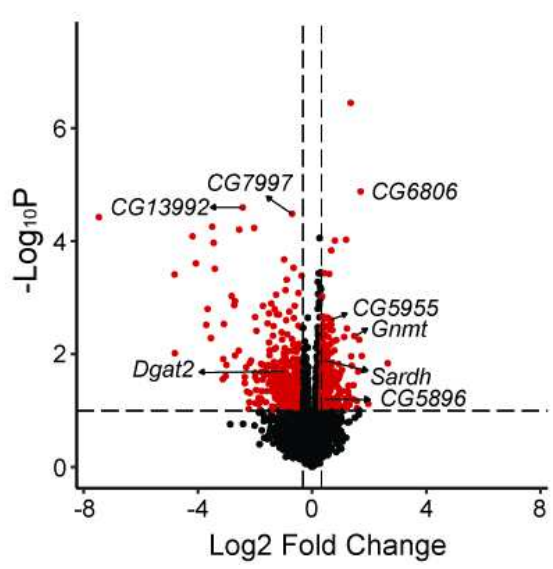

f

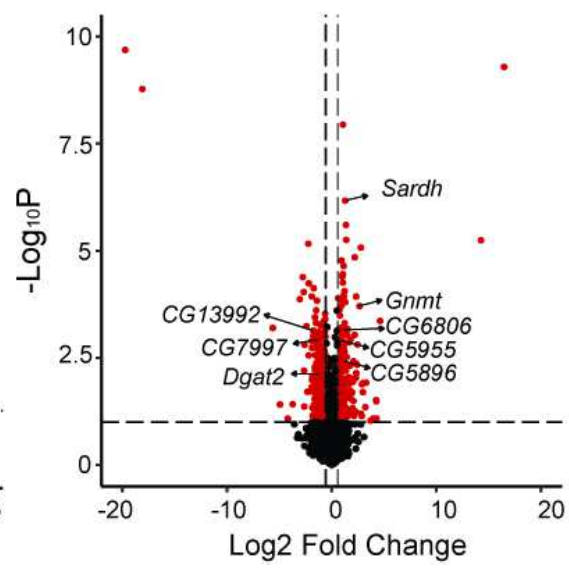

b

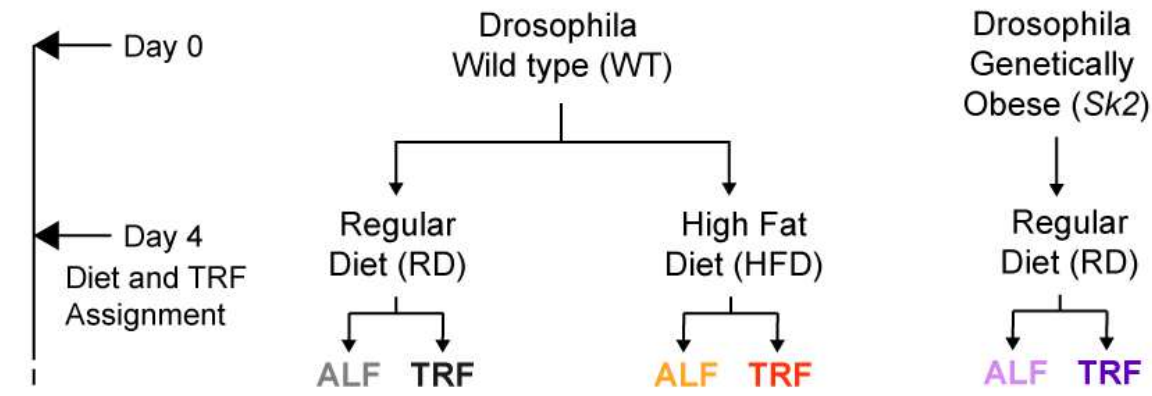

Diet-Induced

Obesity Model

(DIO)

Genetic-Induced

Obesity Model

(GIO)

d Down Genes under TRF

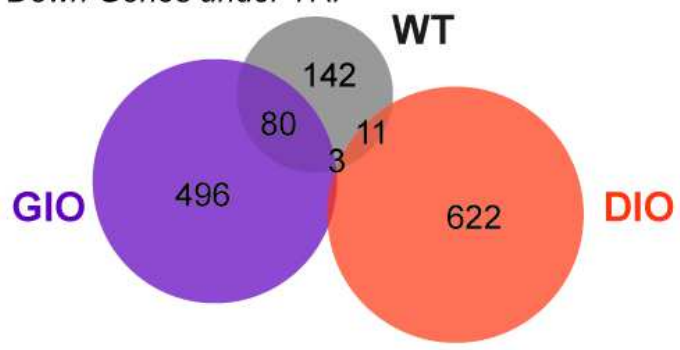

g

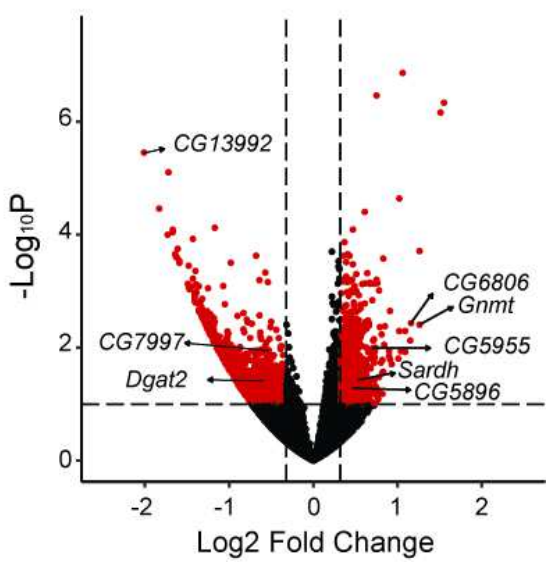

Figure 1

Common differentially expressed genes identified under TRF versus ALF in WT and obesity models. (a) Schematic depicting the timing of food access in ALF (ad libitum feeding) and TRF (time-restricted feeding). In ALF, flies have unrestricted access to food. In TRF, flies could consume food only during the 
$12 \mathrm{~h}$ of daytime. (b) Flow chart depicting the experimental set up. Wild type and genetic obese mutant Sk2 flies were separated into respective food conditions regular diet (RD) or high fat diet (HFD) to establish WT, GIO and DIO models. Fly models were further separated into either ALF or TRF. Thoraces were collected at 3 weeks of age every 4 hours totaling to 6 time points for RNA sequencing. (c) Venn diagram of significantly upregulated genes performed using DeSeq2 ( $p$ value $\leq 0.05$, Fold change $\geq 1.25$ ) under TRF in WT, DIO and GIO models. (d) Venn diagram of significantly downregulated genes using DeSeq2 ( $p$ value $\leq 0.05$, Fold change $\geq 1.25$ ) under TRF in WT, DIO and GIO models. (e-g) Volcano plots showing expression profiles of common DEGs under TRF versus ALF in WT (e), DIO (f) and GIO (g) models. Red dots represent differentially expressed genes under TRF ( $p$ value $\leq 0.05$, Fold change $\geq 1.25$ ). 
Figure 2

a

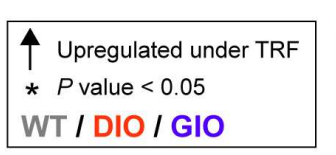

C
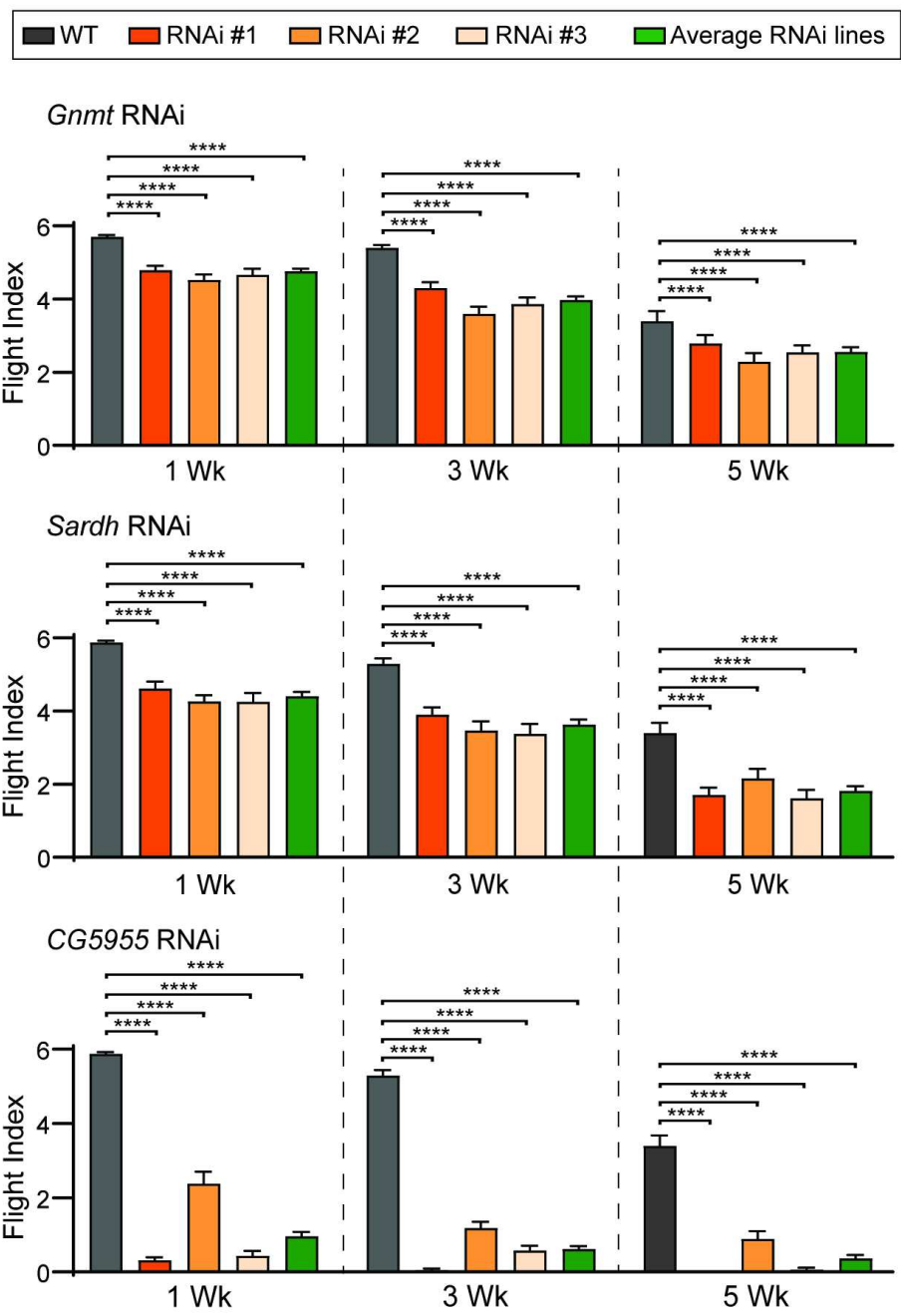

b

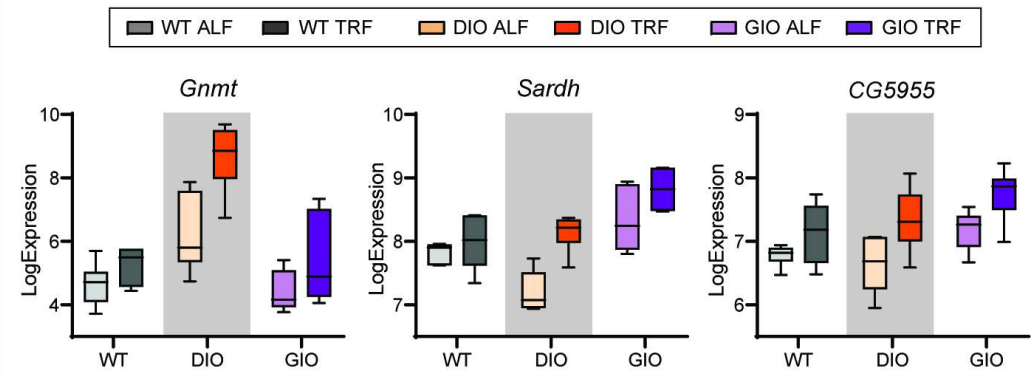

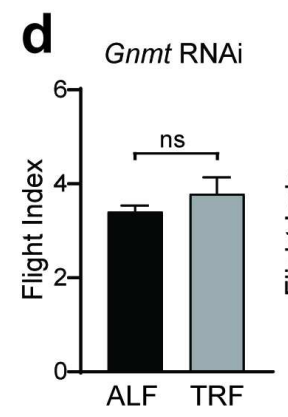
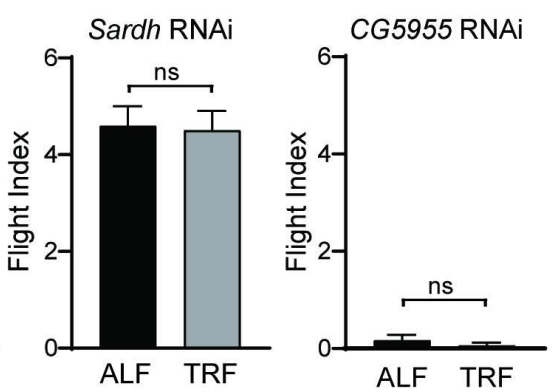

e
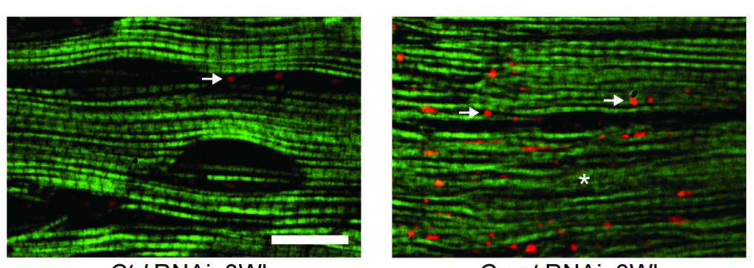

Ctrl RNAi, 3Wk

Gnmt RNAi, 3Wk
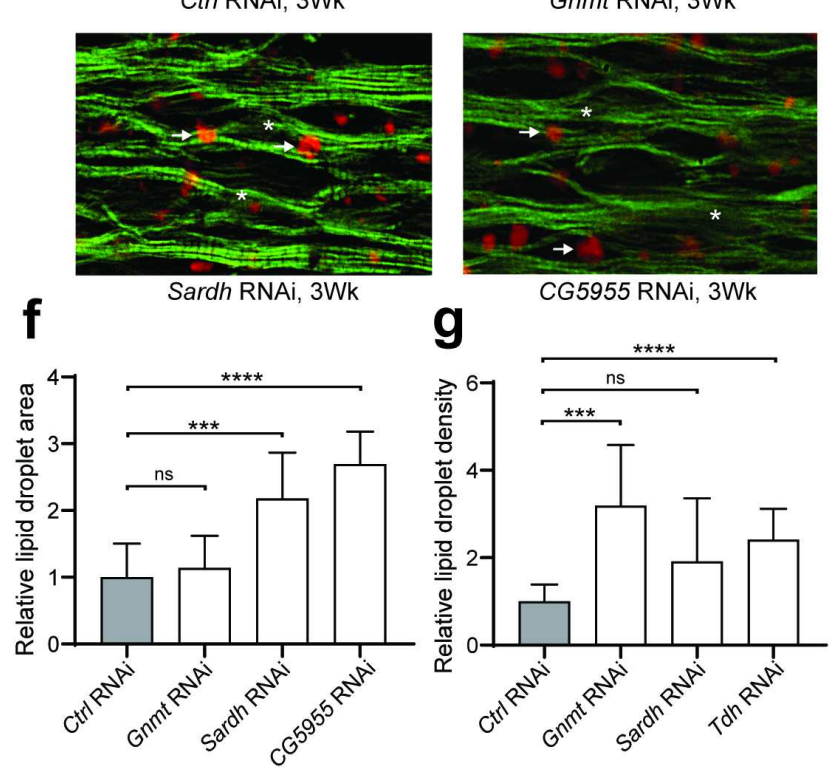

g

CG5955 RNAi, 3Wk

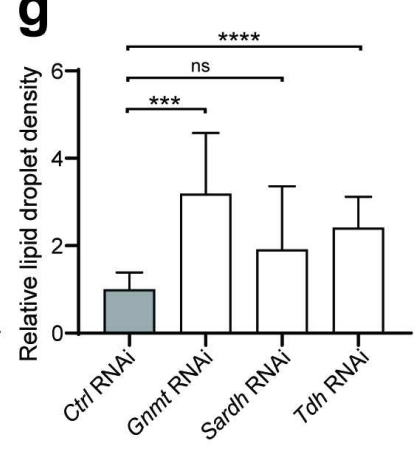

Figure 2

Gene expression and functional validation of Gnmt, Sardh and CG5955 in skeletal muscle in WT and obesity models. (a) Schematic representation of Gnmt, Sardh and CG5955 connection with glycine utilization and production with metabolite intermediates and important genes (italic bold) encoding enzymes. Up arrows indicate upregulation of gene expression (fold change $\geq 1.25$ ) and color indicates condition (grey WT, Orange DIO, purple GIO). Asterisk indicates $p$ value $\leq 0.05$ and fold change $\geq 1.25$. (b) 
Expression level (normalized read count, log2) of gene Gnmt, Sardh and CG5955 under ALF and TRF in WT, DIO, GIO models. (c) Flight performance of WT female flies with Gnmt, Sardh or CG5955 IFM-specific KD at 1 week, 3 week and 5 weeks of age. Three independent RNAi lines per gene were tested and plotted

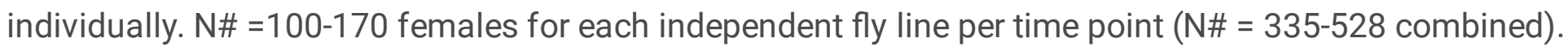
(d) Flight performance of KD in common upregulated genes at 3 weeks of age unchanged in TRF condition. As the three independent lines showed similar results in (c), one fly line from an independent

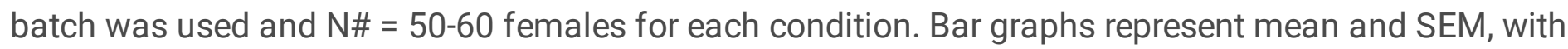
significance presented as $n s p>0.05,{ }^{*} p \leq 0.05,{ }^{*} p<0.01,{ }^{\star \star \star} p<0.001$. (e) Fluorescence images of the IFM from 3-week-old females of control and IFM-specific KD of Gnmt, Sardh and CG5955 flies upon probing with phalloidin (green) and Nile Red (red puncta). More accumulation of lipid (arrows) and actincontaining myofibrillar disorganization was detected in IFM of KD of Gnmt, Sardh and CG5955 (asterisks), compared with age-matched control. Scale bar is $20 \mu \mathrm{m}$. (f-g) Intramuscular lipid quantification (size (f) and density (g)) showed significant increase upon IFMspecific KD of Gnmt, Sardh and CG5955 compare to age-matched control. N\# = 9 from three flies' IFM per genotype. 


\section{Figure 3}

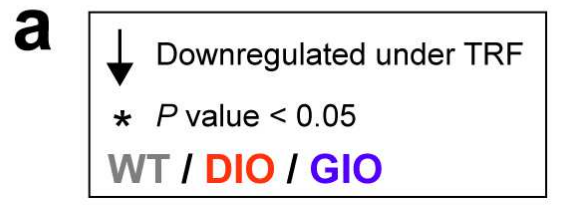

G3P
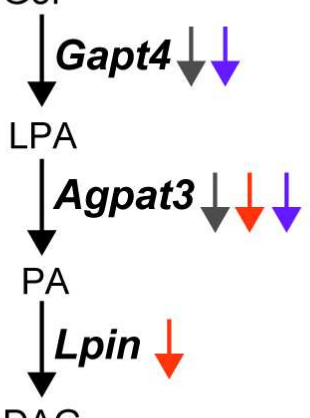

DAG
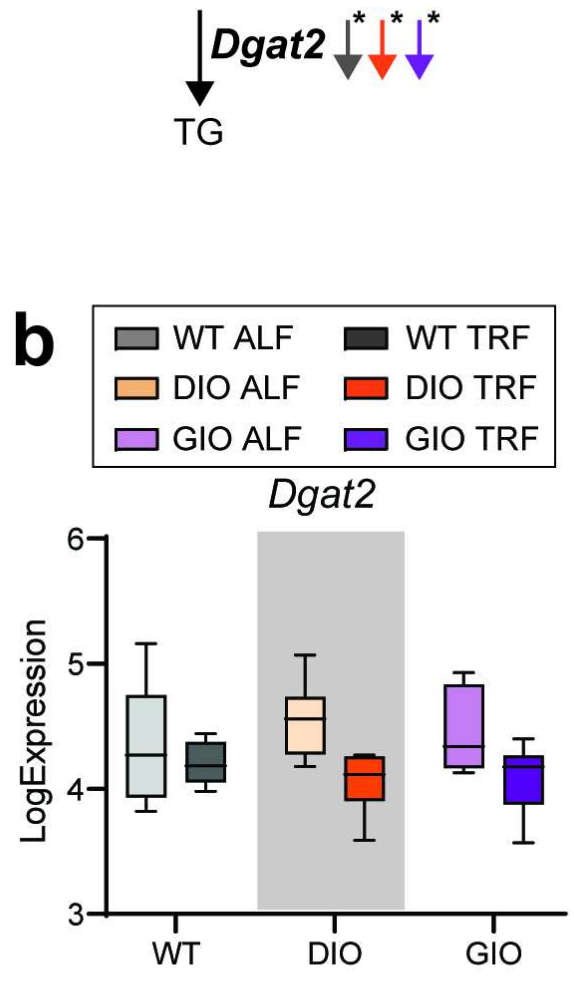
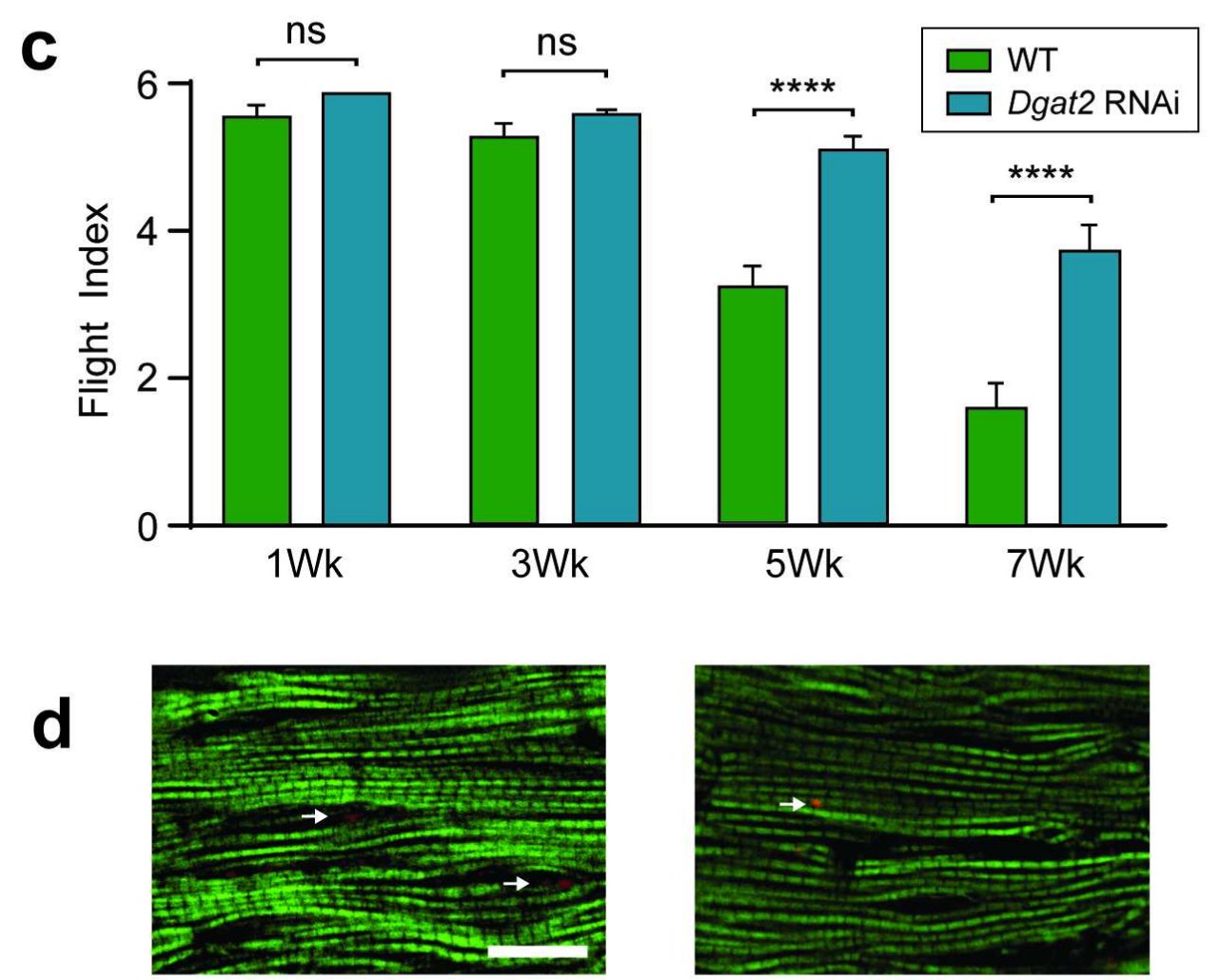

Ctrl RNAi, 3Wk

e

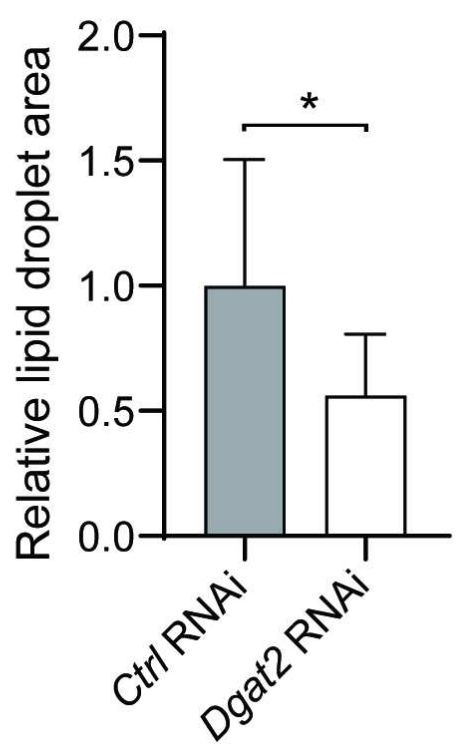

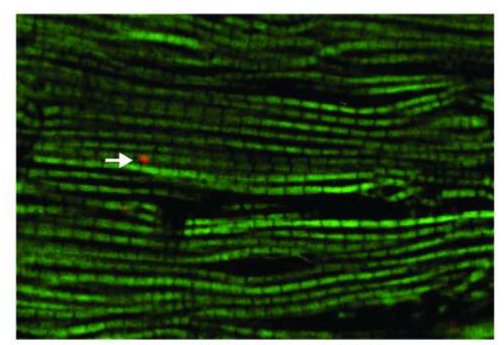

Dgat2 RNAi, 3Wk

f

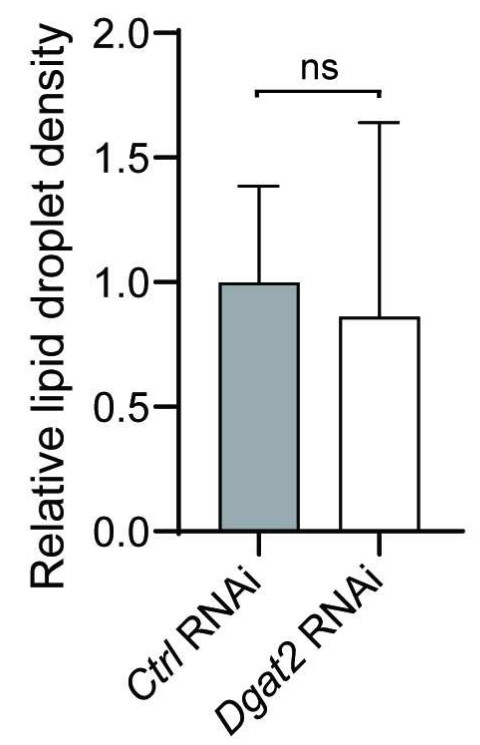

Figure 3

Gene expression and functional validation of Dgat2 in skeletal muscle in WT and obesity models. (a) Schematic representation of de novo triacylglycerol synthesis with metabolite intermediates and important genes (italic bold) encoding enzymes. Down arrows indicate downregulation of gene expression and color indicates condition (grey WT, Orange DIO, purple GIO). Asterisk indicates p-value $<0.05$ and fold change $>1.25$. (b) Expression level (normalized read count, log2) of gene Dgat2 under ALF 
and TRF in WT, DIO, GIO models. (c) Flight performance of WT female flies with Dgat2 KD at 1, 3, 5 and 7 weeks of age. $\mathrm{N \#}=65-88$ female flies per time point. Bar graphs represent mean and SEM, with significance presented as $n s p>0.05,{ }^{\star} p \leq 0.05,{ }^{*} p<0.01,{ }^{\star \star \star} p<0.001$. (d) Fluorescence images of the IFM from 3-week-old females with IFM-specific KD of Dgat2 upon probing with phalloidin (green) and Nile Red (red puncta). Smaller lipid (arrows) was detected in IFM of KD of Dgat2 (asterisks), compared with age-matched control. Scale bar is $20 \mu \mathrm{m}$. (e-f) Intramuscular lipid quantification (size (e) and density (f)) showed significant reduction in lipid droplet area upon IFM-specific KD of Dgat2 compare to age-matched control. $\mathrm{N \#} \mathrm{=} 9$ from three flies' IFM per genotype. 


\section{Figure 4}
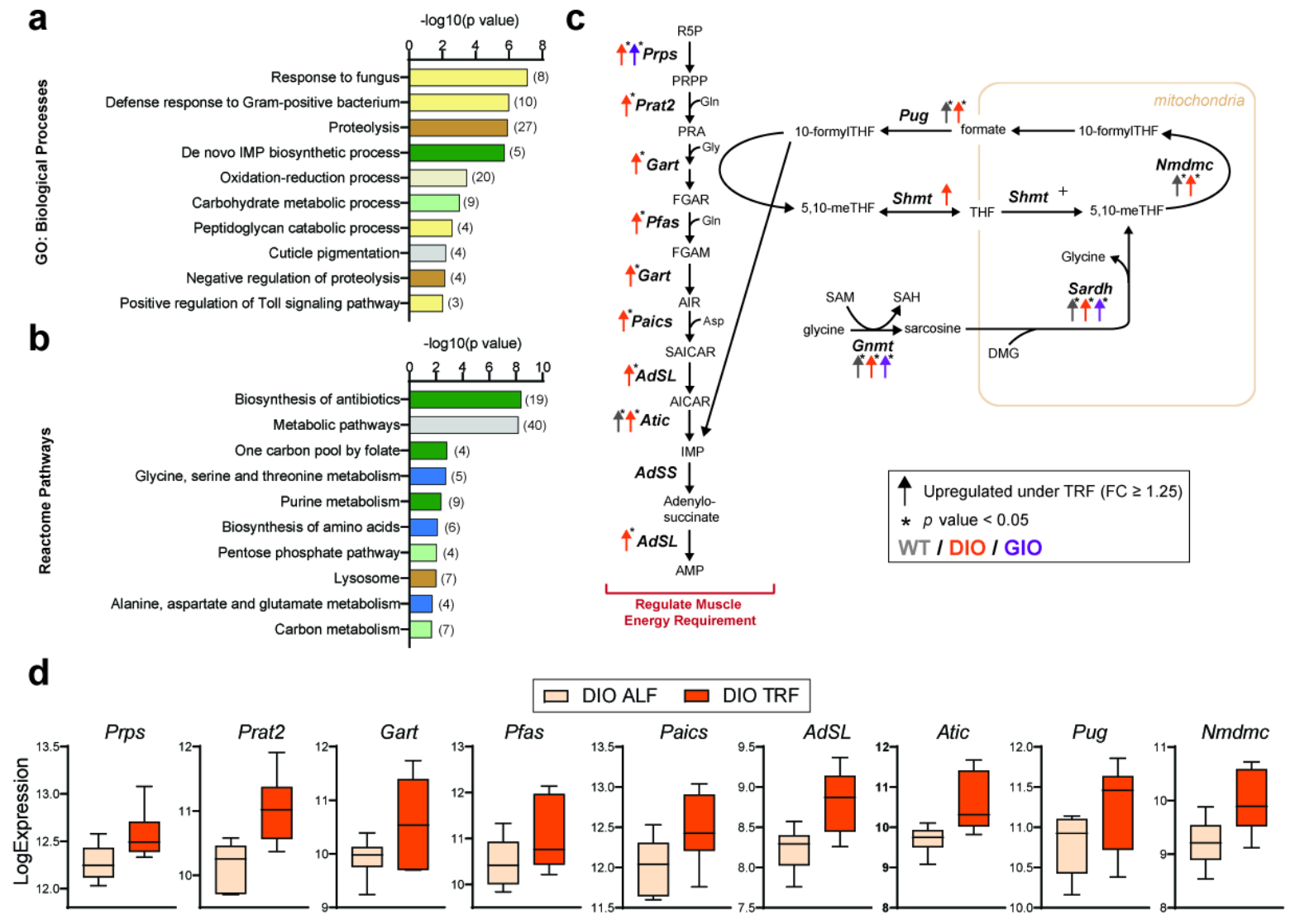

e

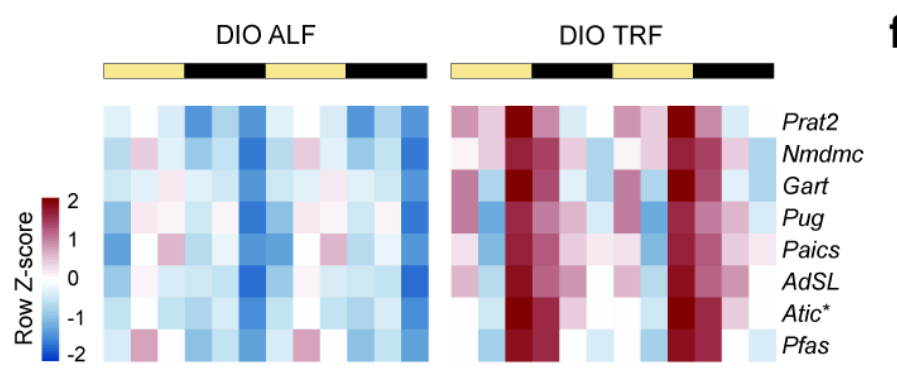

g
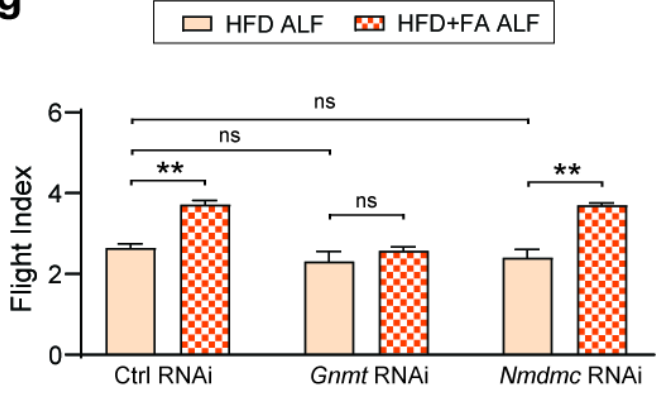

h
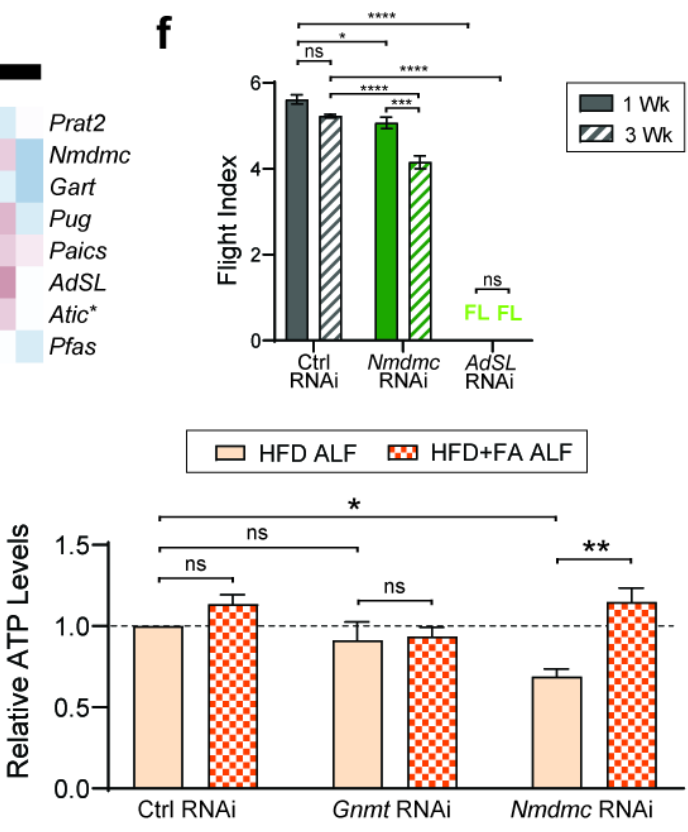

\section{Figure 4}

TRF upregulated genes linked with purine biosynthesis and folate cycle in DIO model. (a) GO analysis and (b) Reactome pathway analysis of 270 genes that were significantly upregulated under TRF in the DIO model. Bar charts represent the - $\log 10$ ( $p$ value) of each enriched GO term and pathway. Related pathways are colored similarly. The number of genes identified in each GO term and pathway is shown in parenthesis. (c) Schematic representation of de novo purine biosynthesis and folate cycle with metabolic 
intermediates and important genes (italic bold) encoding enzymes possibly involved in energy regulation in muscle. Up arrows indicate upregulation of gene expression (fold change $\geq 1.25$ ), asterisk indicates $p$ value $\leq 0.05$ and color indicates condition (grey WT, Orange DIO, purple GIO). (d) Expression level (normalized read count, log2) of significantly upregulated genes in de novo purine biosynthesis and folate cycle under TRF versus ALF in the DIO model. (e) Heatmap representation of the genes in (d) with periodicity in the DIO model. Asterisk indicates the gain of periodicity under TRF. (f) Flight performance of control, Nmdmc KD and AdSL KD at 1 and 3 weeks of age. FL indicates flightless. N\# = 50-70 per condition. FL indicates flightless. Error bars represent SEM. Bar graphs show mean and SEM, with significance presented as nsp $>0.05,{ }^{\star} p \leq 0.05,{ }^{\star} p<0.01$, ${ }^{\star \star \star} p<0.001$. (g) Flight performance of control, Gnmt KD and Nmdmc KD at 3 weeks of age under HFD ALF, and HFD+FA ALF. Error bars represent SEM. $\mathrm{N} \#$ 40-92 per condition. Bar graphs show mean and SEM, with significance presented as nsp $>0.05$, * $\mathrm{p} \leq$ $0.05,{ }^{\star \star} \mathrm{p}<0.01,{ }^{* \star *} \mathrm{p}<0.001$. (h) Relative ATP level measurement in control, IFM-specific KD of Gnmt and Nmdmc at 3 weeks of age under HFD ALF and HFD+FA ALF conditions with N\# = 3-5 per condition. Bar graphs represent mean and SEM, with significance presented as nsp $>0.05,{ }^{*} p \leq 0.05,{ }^{*} p<0.01$, $* \star * p<$ 0.001 . 
Figure 5

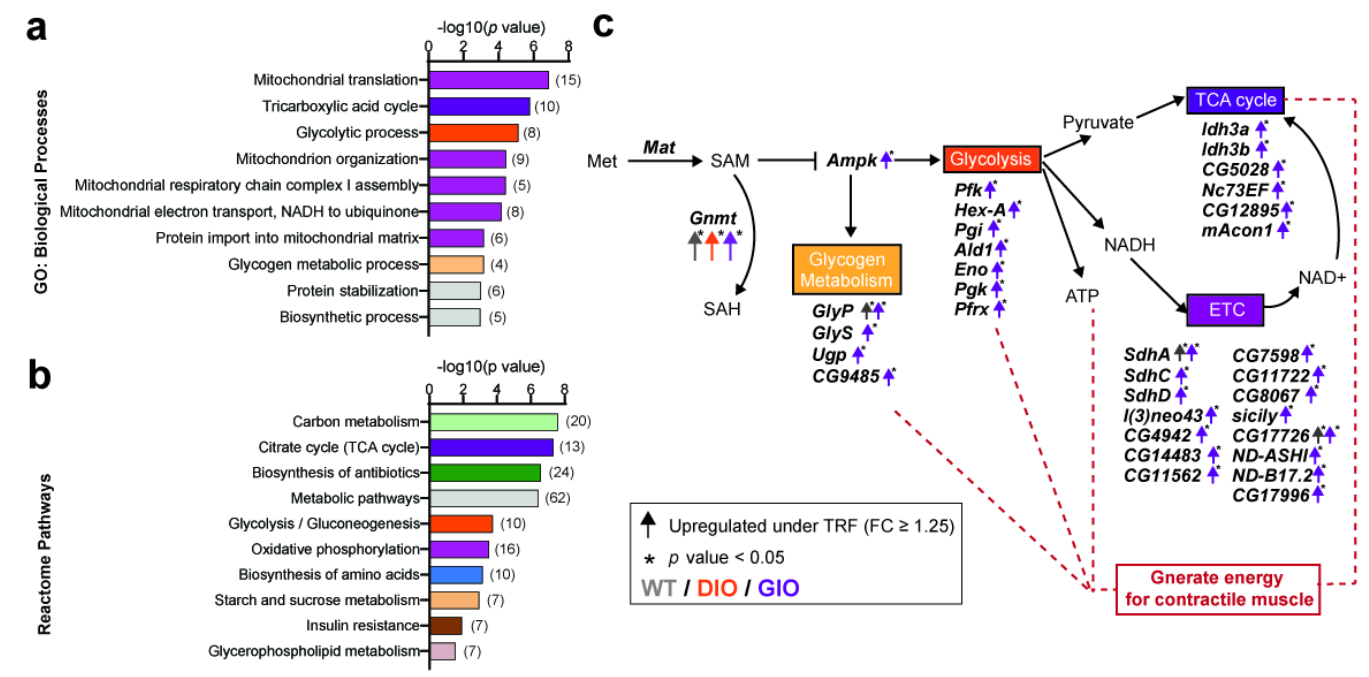

d
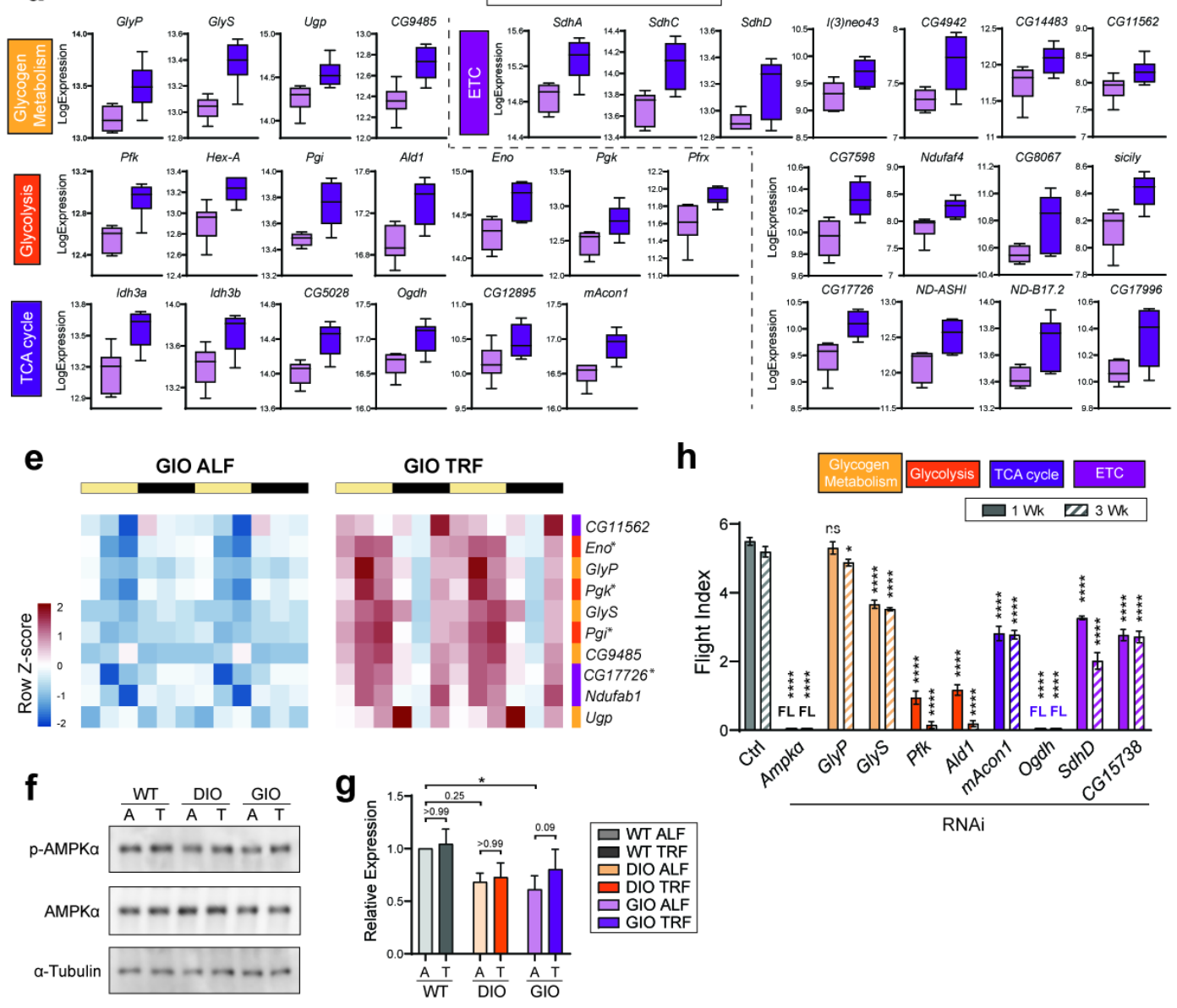

Figure 5

TRF upregulated genes associated with AMPK and downstream pathways in the GIO model. (a) GO analysis and (b) Reactome pathway analysis of 408 genes that were significantly upregulated under TRF in the GIO model. Bar charts represent the - $\log 10$ ( $p$ value) of each enriched GO term and pathway. Related pathways are colored similarly. The number of genes identified in each GO term and pathway is shown in parenthesis. (c) Schematic representation of connection between Gnmt, Ampk and downstream 
of AMPK signaling with important genes (italic bold) encoding enzymes. Up arrows indicate upregulation of gene expression (fold change $\geq 1.25$ ) and color indicates condition (grey WT, Orange DIO, purple GIO). Asterisk indicates $p$ value $\leq 0.05$ and fold change $\geq 1.25$. The up arrow of Ampk represents the expression of AMPK a subunit. (d) Expression level (normalized read count, log2) of significantly upregulated genes in glycolysis, glycogen metabolism, TCA cycle and electron transport chain under TRF versus ALF in the GIO model. (e) Heatmap representation of the genes in (d) with periodicity in the GIO model. Asterisk indicates the gain of periodicity under TRF. (f) Representative western blot of p-AMPKa levels (top), AMPKa levels (middle) and a-tubulin (bottom), from 3-week-old female fly IFMs in WT, DIO and GIO models under ALF (A) and TRF (T). (g) Ratios of p-AMPKa/AMPKa normalized to WT ALF with N\# = 3. (h) Flight index of GIO genes in relation to downstream pathways shown at 1 and 3 weeks of age. $\mathrm{N \#} \mathrm{=} \mathrm{50-}$ 100 per condition. FL indicates flightless. Bar graphs represent mean and SEM, with significance presented as nsp $>0.05,{ }^{*} p \leq 0.05,{ }^{* *} p<0.01,{ }^{* * *} p<0.001$.

\section{Figure 6}

\section{Diet-Induced Obesity (DIO)}

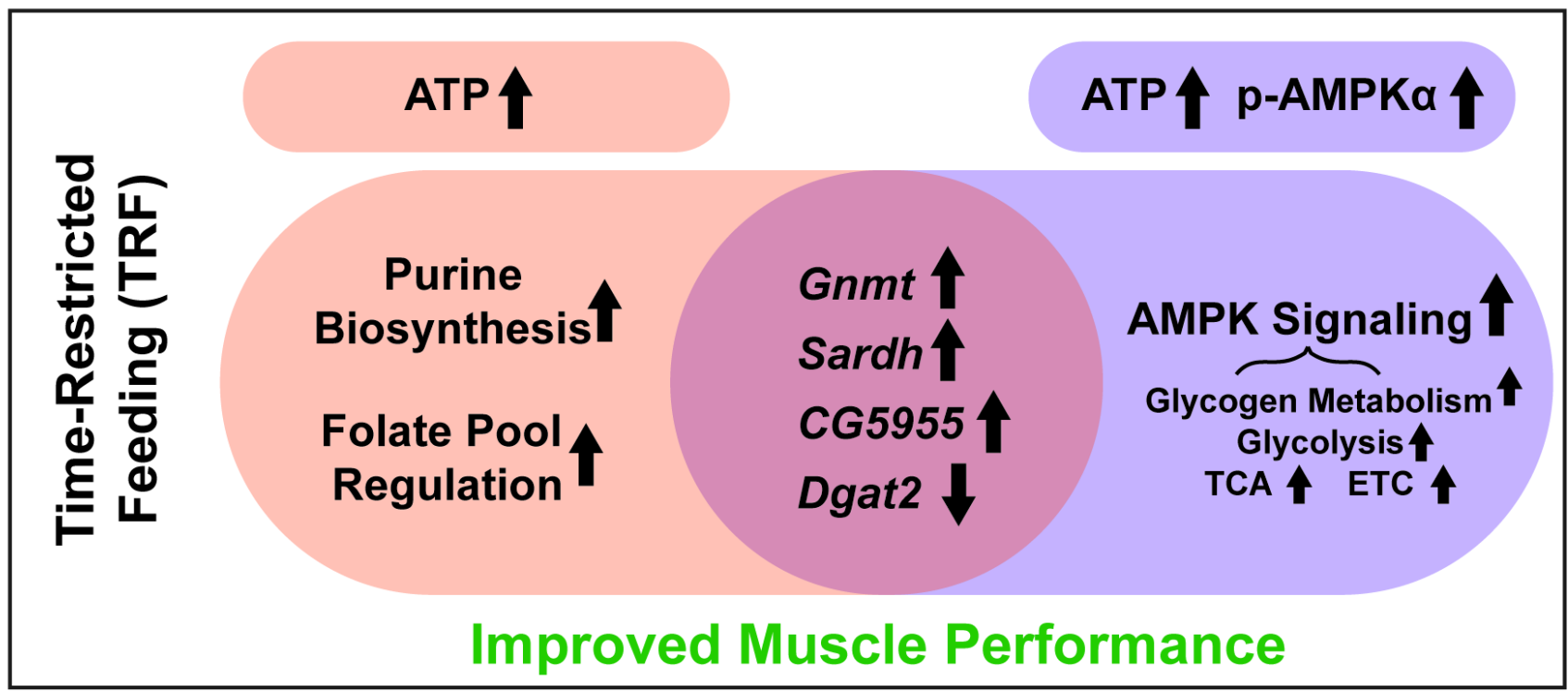

Figure 6

Proposed mechanism of TRF in Drosophila skeletal muscle under obesogenic challenges. $12 \mathrm{~h}$ eating and $12 \mathrm{~h}$ fasting TRF intervention led to increased SAM regulation by upregulating of Gnmt, Sardh and CG5955, along with decreased TAG synthesis, which is supported by downregulation of Dgat2 in all conditions. In the diet-induced obesity model, TRF promoted purine biosynthesis and folate pool regulation, potentially resulting in higher ATP levels. However, in the genetic-induced obesity model, AMPK signaling, and downstream pathways were exclusively upregulated via increased p-AMPKa levels and increased ATP levels. All pathways regulated led to improved muscle performance in obesity models. 


\section{Supplementary Files}

This is a list of supplementary files associated with this preprint. Click to download.

- SupplementaryMaterialsTRFMuscleTranscriptomicsManuscript.pdf

- SupplementaryTables18TRFMuscleTranscriptomicsManuscript.xIsx 